\title{
Thermodynamic evolution of the cosmological baryonic gas
}

\section{Galaxy formation ${ }^{\star}$}

\author{
J.-M. Alimi ${ }^{1}$ and S. Courty ${ }^{1,2}$ \\ 1 Laboratoire de l'Univers et de ses Théories, CNRS UMR 8102, Observatoire de Paris-Meudon, 5 place Jules Janssen, \\ 92195 Meudon, France \\ e-mail: jean-michel.alimi@obspm.fr \\ 2 Present address: Science Institute, University of Iceland, Dunhagi 3, 107 Reykjavik, Iceland \\ e-mail: courty@raunvis.hi.is
}

Received 9 April 2004 / Accepted 25 November 2004

\begin{abstract}
The problem of galaxy formation and its dependence on thermodynamic properties is addressed by using Eulerian hydrodynamic numerical simulations of large scale structure formation. Global galaxy properties are explored in simulations including gravitation, shock heating and cooling processes, and following self-consistently the chemical evolution of a primordial composition hydrogen-helium plasma without assuming collisional ionization equilibrium. The galaxy formation model is mainly based on the identification of converging dense cold gas regions. We show that the evolution at low redshift of the observed cosmic star formation rate density is reproduced, and that the galaxy-like object mass function is dominated by low-mass objects. The galaxy mass functions are well described by a two power-law Schechter function whose parameters are in good agreement with observational fits of the galaxy luminosity function. The high-mass end of the galaxy mass function includes objects formed at early epochs and residing in high-mass dark matter halos whereas the low-mass end includes galaxies formed at later epochs and active in their "stellar" mass formation. Finally, the influence of two other physical processes, photoionization and non-equipartition processes between electrons, ions and neutrals of the cosmological plasma is discussed and the modifications on galaxy formation are examined.
\end{abstract}

Key words. cosmology: theory - large-scale structure of the Universe - intergalactic medium - galaxies: formation hydrodynamics - galaxies: luminosity function, mass function

\section{Introduction}

Processes like gravitation, shock heating, radiative cooling, photoionization and non-equipartition, among others, play a crucial role in the evolution of the thermodynamic properties of baryonic matter. As galaxies originate in cold and dense gas regions, any change in the gas thermodynamic properties should have an effect on their formation. This connection is the issue here addressed. Numerical simulations have the significant advantage of being able to include a large set of physical processes involved in galaxy formation. Even if a phenomenological description of this process needs to be adopted, global galaxy properties are now extensively studied, like the cosmic star formation rate density, the galaxy mass/luminosity function, the clustering properties, etc. Such results use semi-analytical approaches (Somerville \& Primack 1999; Cole et al. 2000); hybrid approaches, combination of semi-analytical and $N$-body methods (Kauffmann et al. 1999; Hatton et al. 2003); and hydrodynamical $N$-body

* Appendices are only available in electronic form at http://www. edpsciences.org simulations using Smooth-Hydrodynamic-Particle (Pearce et al. 2001; Weinberg et al. 2002), Lagrangian (Gnedin 1996) or Eulerian (Cen 1992) methods. These complementary computations are all based, but now in a sophisticated way, on the fundamental ideas that galaxy formation results from the gas accretion and its cooling (Sunyaev \& Zel'dovich 1972; Rees \& Ostriker 1977; Silk 1977; White \& Rees 1978) into a network of overdense structures created by the gravitating dark matter on the large scales of the universe. Moreover, quantities computed from numerical simulations can now be compared with the huge amount of available observational data. The galaxy luminosity function (Binggeli et al. 1988) is widely estimated, in different surveys, towards fainter magnitude, in several wavelength bands, and for different classes of galaxies (Madgwick 2002). Nevertheless some discrepancies remain: Fig. 1 in Cross et al. (2001) shows a dispersion of a factor of 2 at the characteristic luminosity, $L_{*}$, and a factor of 10 at $0.01 L_{*}$. A number of studies attempt to retrieve the galaxy mass function from the galaxy luminosity function by using stellar population synthesis models. Numerical simulations take the opposite approach: their first output is mass and by using stellar population 
synthesis models they can provide luminosity. Here we focus, among other things, on the cosmological evolution of the galaxy mass function and examine in detail its shape at $z=0$ and $z=1$. We then derive mass-to-luminosity ratios to compare with the observed galaxy luminosity function.

As this paper is the second in a series of three, focusing on the influence of thermodynamics on galaxy formation, the purpose is not to compute sophisticated models of galaxy formation but rather emphasize the description of cosmological gas. Hence to keep the model free of parameters as much as possible, we only consider in the simulations the dominant physical processes: gravitation, shock heating, radiative cooling, but neglect e.g. feedback processes. A model of galaxy formation is also introduced. The first part of the paper examines global galaxy properties, the cosmic star formation rate density, the galaxy mass function and the epoch of formation. The main results are the following: 1. Galaxy formation is a hierarchical process mainly driven by the amount of available cold gas in the inter-galactic medium; 2 . the majority of the high-mass galaxies form at early epochs; 3 . the galaxy population at any given redshift is dominated by a significant fraction of low-mass galaxies formed at early as well as late epochs. The halo dark matter mass function is also explored and a preliminary study of the galaxy distribution inside halos is presented. The second part of the paper analyzes separately the influence of photoionization, from ultraviolet background radiation, and the influence of non-equipartition processes between ions, neutrals and electrons of the cosmological plasma. Nonequipartition has been scrutinized in Courty \& Alimi (2004) (Paper I) using two numerical simulations: the first one taking into account non-equipartition processes and denoted by $S_{3 \mathrm{~T}}$ and the second one, denoted by $S_{1 \mathrm{~T}}$, in which equipartition between species is forced. The former simulation allows each species to carry its own internal energy whereas the latter one assumes that ions, neutrals and electrons have the same temperature. That paper concludes that a significant fraction of the inter-galactic medium (the plasma inside gravitationally bound structures), that is accreted in not too dense structures and at temperatures in the range $10^{4}-10^{6} \mathrm{~K}$, is out of equilibrium and warmer in the $S_{3 \mathrm{~T}}$ than in the $S_{1 \mathrm{~T}}$ simulation. Nonequipartition processes are likely to be dominant before the end of the reionization epoch. As galaxies are accreting their gas from the inter-galactic medium in the temperature range $10^{4}-10^{6} \mathrm{~K}$, this implies an influence of the non-equilibrium thermodynamics on the galaxy formation process. Quantifying this change is one of the purposes of this paper. The third paper in this series (Courty \& Alimi, in preparation), will quantify how galaxy clustering properties and the cosmological bias are modified.

This paper is organized as follows. Numerical simulations and the galaxy formation model are described in Sect. 2. Section 3 presents galaxy properties: the cosmic star formation rate density, the galaxy-like object mass function, and the epoch of formation. The dark matter halo mass function and the galaxy distribution inside the biggest mass halos are discussed. Fits of the galaxy-like object and dark matter halo mass functions are given. The influence of photoionization and non-equipartition processes are shown in Sects. 4 and 5, respectively. Conclusions are given in Sect. 6.

\section{Numerical simulations}

The simulations were performed with a 3 dimensional $\mathrm{N}$-body/hydrodynamical code, coupling a Particle-Mesh method for computing gravitational forces with a Eulerian method (Teyssier et al. 1998). The simulations include shock heating, radiative cooling, photoionization processes, nonequipartition processes between the ions, electrons and neutrals of the cosmological plasma, and galaxy formation. The features of the simulations, analyzed here, are the following: the $G 0$ and $G 1$ simulations include shock heating, radiative cooling and galaxy formation. They only differ in the resolution. In addition to these processes, the $G P$ simulation includes photoionization processes and GNE includes non-equipartition processes but not photoionization. We refer to Paper I for details about shock heating treatment, non-equipartition processes and the radiative cooling terms. These latter terms include collisional excitation, collisional ionization, recombination $^{1}$, bremsstrahlung and Compton scattering. To use the same notations as in Paper I, $G 0, G 1$ and $G P$ are $S_{1 \mathrm{~T}}$ simulations, in which equipartition between species (ions, neutrals and electrons) is forced and the cosmological plasma has a single temperature. The GNE simulation is a $S_{3 \mathrm{~T}}$ simulation with each species having its own internal energy.

The GP simulation takes into account the ionization and heat input from an ultraviolet background radiation to reproduce conditions after the reionization epoch. The photoionization and heating rates are computed from the evolution of the hydrogen and helium densities and from the spectrum of the ultraviolet background radiation $J(v)$. The radiation is considered a spatially uniform field over the computational volume. The density evolution equations, Eqs. (5) to (7) in Paper I, are now:

$$
\begin{aligned}
-\beta_{\mathrm{H}^{0}} n_{\mathrm{e}} n_{\mathrm{H}^{0}}+\alpha_{\mathrm{H}^{+}} n_{\mathrm{e}} n_{\mathrm{H}^{+}}-\Gamma_{\mathrm{H}^{0}} n_{\mathrm{H}^{0}} & =\frac{\partial n_{\mathrm{H}^{0}}}{\partial t} \\
-\beta_{\mathrm{He}^{0}} n_{\mathrm{e}} n_{\mathrm{He}^{0}}+\alpha_{\mathrm{He}^{+}} n_{\mathrm{e}} n_{\mathrm{He}^{+}}-\Gamma_{\mathrm{He}^{0}} n_{\mathrm{He}^{0}} & =\frac{\partial n_{\mathrm{He}^{0}}}{\partial t} \\
\beta_{\mathrm{He}^{+}} n_{\mathrm{e}} n_{\mathrm{He}^{+}}-\alpha_{\mathrm{He}^{++}} n_{\mathrm{e}} n_{\mathrm{He}^{++}}+\Gamma_{\mathrm{He}^{+}} n_{\mathrm{He}^{+}} & =\frac{\partial n_{\mathrm{He}^{++}}}{\partial t}
\end{aligned}
$$

where $n_{\mathrm{H}^{0}}, n_{\mathrm{H}^{+}}, n_{\mathrm{He}^{0}}, n_{\mathrm{He}^{+}}, n_{\mathrm{He}^{++}}$and $n_{\mathrm{e}}$ are the six densities of the primordial composition hydrogen-helium cosmological plasma, and $\beta_{i}$ and $\alpha_{i}$ are the ionization and recombination rates (given in Table 1 in Paper I). Photo-ionization rates $\Gamma_{i}$ are expressed by:

$\Gamma_{i}=\int_{v_{i}}^{\infty} \frac{4 \pi J(v)}{h v} \sigma_{i}(v) \mathrm{d} v$

\footnotetext{
1 The helium recombination rate of these simulations has an incorrect temperature dependence, although the expression for $\alpha_{\mathrm{H}_{\mathrm{e}}^{++}}$in Table 1 of Paper I is commonly used in the literature. The correct expression should involve the nuclear charge of the helium atoms (see Spitzer 1978): $\alpha_{\mathrm{H}_{\mathrm{e}}^{++}}=3.36 \times 10^{-10}\left(1+\left(T_{\mathrm{e} 6} / 4\right)^{0.7}\right)^{-1} T_{\mathrm{e}}^{-1 / 2} T_{\mathrm{e} 3}^{-0.2}$. Since collisional excitation is the dominant net cooling term this mistake should have a limited effect on the results.
} 


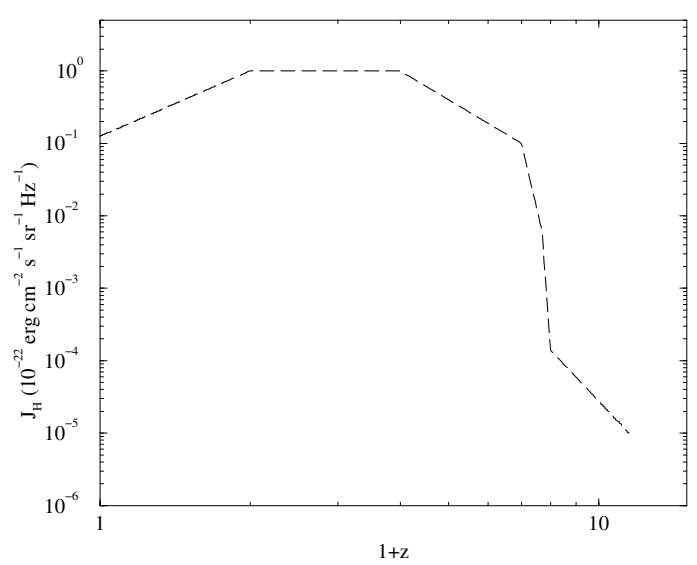

Fig. 1. Evolution with redshift of the ultraviolet background radiation intensity.

with $i$ denoting the species $\mathrm{H}^{0}, \mathrm{He}^{0}, \mathrm{He}^{+}, \sigma_{i}$ is the effective cross-section for species $i$ (taken from Osterbrock 1989), $h v_{i}$ is the ionization energy for the species $i$, and $J(v)$ is the background radiation intensity. The photoionization processes are also a heating source (see Eq. (4) in Paper I) and the heating rates are expressed by:

$\mathrm{H}_{i}=n_{i} \int_{v_{i}}^{\infty} \frac{4 \pi J(v)}{h v} \sigma_{i}(v)\left(h v-h v_{i}\right) \mathrm{d} v$.

The shape of the background intensity spectrum is defined by a function $F(z)$ characterizing the evolution with redshift of the ultraviolet background radiation (Katz et al. 1996):

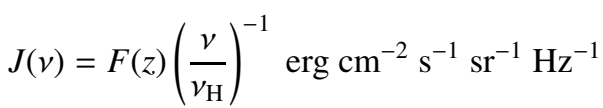

where $h v_{\mathrm{H}}=13.598 \mathrm{eV}$ is the hydrogen ionization threshold. Since this evolution is little-known at high redshift, we estimate $J(v)$ from observational measurements (Scott et al. 2002) and from numerical works (Gnedin 2000). This includes the decline of the ultraviolet background radiation intensity observed between $z \sim 1$ and $z=0$, the sharp increase before $z \sim 6$ and the shallow evolution before $z \sim 7$. We start the reionization at redshift 10.5 with the bulk of the transition between $z=7$ and $z=6$. The function $F(z)$ is plotted in Fig. 1 and its expression, in units of $J_{0}=10^{-22} \mathrm{erg} \mathrm{cm}^{-2} \mathrm{~s}^{-1} \mathrm{sr}^{-1} \mathrm{~Hz}^{-1}$, is:

$F(z)= \begin{cases}10^{(A 1 \log (1+z)+B 1)} & 11.5 \geq 1+z>8 \\ 10^{(A 2 \log (1+z)+B 2)} & 8 \geq 1+z>7.7 \\ 10^{(A 3 \log (1+z)+B 3)} & 7.7 \geq 1+z>7 \\ J_{0}(4 / 1+z)^{4.11} & 7 \geq 1+z>4 \\ J_{0} & 4 \geq 1+z>2 \\ J_{0}(2 /(1+z))^{-3} & 2 \geq 1+z\end{cases}$

with $A 1=-7.27, A 2=-98.32, A 3=-29.52, B 1=$ $-19.28, B 2=62.94, B 3=1.94$.

As it is useful for the discussion in this paper, we now compare the gas distribution in the three kinds of simulations. Figure 2 illustrates the baryonic mass fraction in temperaturedensity diagrams; the top and bottom panels are extracted from Paper I. The top panel is computed with a simulation including hydrodynamical shocks and radiative cooling. The middle
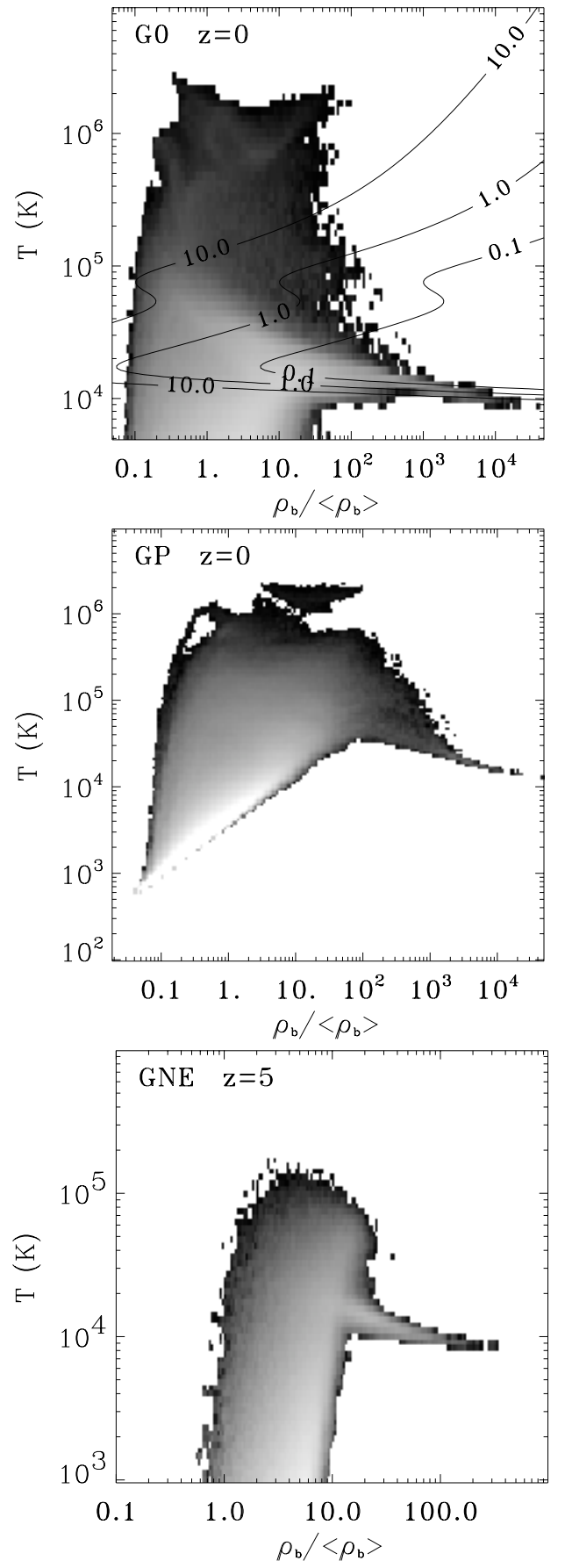

Fig. 2. Isocontours of the baryonic mass fraction per interval of the baryonic density contrast and per interval of temperature computed for the $G 1$ and $G P$ simulations at $z=0$ and for $G N E$ at $z=5$ (top, middle and bottom panels, respectively). The increase in the mass fraction scales from dark to light. Note that $G 1$ and $G N E$ are, for these plots, computed without galaxy formation. Solid curves on the top panel show the ratios $t_{\text {cool }} / t_{\mathrm{ff}}=0.1,1,10$.

panel includes photoionization processes and shows that they are dominant over cooling processes only in low and middle dense regions (see also Weinberg et al. 1997): low density regions are heated up to temperatures between $10^{3}$ and less than $10^{4} \mathrm{~K}$. The competition between photoionization heating and cooling due to adiabatic expansion results in the concentration 
of the gas on a slope $T=T_{0}(\rho / \bar{\rho})^{\gamma}$ with $T_{0} \sim 6 \times 10^{3} \mathrm{~K}$ and $\gamma$ around 0.6 (Katz et al. 1996; Hui \& Gnedin 1997). The bottom panel displays the gas distribution in the GNE simulation and shows a non-negligible warm gas fraction. As the influence of non-equipartition processes is larger at high redshift, the isocontours are plotted at $z=5$ (see discussion in Paper I).

Although differences exist in the low-density regions, altogether the three gas distribution diagrams show a common feature: a peak in the high density, cold region. This gas being the reservoir for galaxy formation, we thus present in the first part of this paper galaxy properties in a simulation only including the dominant processes involved in galaxy formation, namely hydrodynamical shocks and radiative cooling. The influence of photoionization and non-equipartition processes will be considered in separate sections.

We now turn to the description of the galaxy formation model. Numerical simulations of large scale structure formation currently do not allow for the formation of objects beyond the scale of a grid cell, a few times $10^{6} M_{\odot}$, this mass being much larger than the mass of a single star. The problem is bypassed by considering the physical conditions needed to form a galaxy. The most important condition is that the gas cloud is collapsing, meaning that the cooling time is less than the dynamical time or the free fall time (Rees \& Ostriker 1977). The top panel of Fig. 2 illustrates in a temperature-baryonic density diagram that dense and cold gas regions are located inside the iso-contour $t_{\text {cool }} / t_{\mathrm{ff}}=1$. The galaxy formation model then consists of the identification of the gas satisfying this criteria. A fraction of the baryonic matter is turned into a "stellar" particle describing the amount of stellar mass produced during the process of galaxy formation. Galaxy-like objects are then defined by a collection of this "stellar" mass (the term "galaxylike object" has already been used in Evrard et al. (1994), although describing high-density contrast baryonic clumps). To make sure that gas regions giving birth to galaxies are correctly identified we add other criteria described below. The purpose of this paper being to show how modifications of the gas thermodynamics have an effect on the galaxy formation process, we then deliberately keep the number of free parameters low. This model, although very simple, gives consistent results between the properties of the galaxy-like objects in the simulation and their observational counterparts.

To express the condition $t_{\text {cool }}<t_{\text {ff }}$, we define the cooling timescale $t_{\text {cool }}$, computed from the internal energy variation of the gas $E / \dot{E}$, and the dynamical time or free fall time:

$t_{\mathrm{ff}}=\sqrt{\frac{3 \pi}{32 G \rho}}$.

One of the other conditions requires that the size of the gas cloud must be less than the Jean's length given by:

$\lambda_{\mathrm{J}}=c_{\mathrm{s}}\left(\frac{\pi}{G \rho}\right)^{1 / 2}$.

Note that the total matter density, including dark matter, baryonic matter and "stellar" particles, is used in the expressions of the dynamical time and the Jean's length. It is clear that the Jeans criterion is reliable only down to the mass resolution, since the size of gas clouds itself is limited by the spatial resolution of the simulation. A third condition is that the gas must be in a converging flow: $\nabla \cdot \boldsymbol{v}<0$. Finally the baryonic density contrast, $\delta_{\mathrm{B}} \equiv(\delta \rho / \bar{\rho})_{\mathrm{B}}$, must be higher than a threshold $\left(1+\delta_{B}\right)_{\mathrm{s}}$. This is taken to be the value of the baryonic density contrast at the turnaround, 5.5, computed in the top-hat collapse spherical model (Padmanabhan 1993).

To estimate the amount of "stellar" mass formed, we express the variation of the baryonic mass as the ratio between the available baryonic mass $m_{\mathrm{B}}$ and a characteristic timescale $t_{*}$ :

$$
-\frac{\mathrm{d} m_{\mathrm{B}}}{\mathrm{d} t}=\frac{m_{\mathrm{B}}}{t_{*}}
$$

The integration of this expression on a timestep $\Delta t=t-t_{0}$ gives:

$m_{\mathrm{B}}(t)=m_{\mathrm{B}}\left(t_{0}\right) \exp \left(-\frac{\Delta t}{t_{*}}\right)$

where $m_{\mathrm{B}}\left(t_{0}\right)$ is the baryonic mass initially present. The "stellar" mass formed is then:

$m_{*}=m_{\mathrm{B}}\left(t_{0}\right)-m_{\mathrm{B}}(t) \simeq m_{\mathrm{B}}\left(t_{0}\right) \frac{\Delta t}{t_{*}}$.

Then in each cell, checking the four criteria described above, a fraction of the gas is turned into a "stellar" particle. Each of these particles carries its mass $m_{*}$ and its epoch of formation given by the scale factor $a_{*}$. The mass $m_{*}$ is computed using Eq. (9) with $m_{\mathrm{B}}\left(t_{0}\right)$ the baryonic mass enclosed within the grid cell at each timestep and with the characteristic time $t_{*}=\max \left(t_{\mathrm{ff}}, 10^{8} \mathrm{yr}\right)$. Figure A.1 in Appendix A shows the evolution in redshift of the "stellar" particle mass function for the different simulations: the "stellar" particle mass ranges between a few times $10^{5}$ and $\sim 2 \times 10^{8} M_{\odot}$. The "stellar" particles are involved in the computation of the gravitational potential and their evolution is treated in the same way as the collisionless dark matter.

At any redshift two catalogs of objects are created: one consisting of dark matter halos and one of galaxy-like objects. Halos and galaxies are defined by grouping either dark matter particles or "stellar" particles with a Friend-of-Friend algorithm. This algorithm joins together all particles separated by a distance proportional to the link parameter $\eta$. We take $\eta=0.2$. We exclude from the dark matter halo catalog groups with less than 10 particles. This threshold is denoted $M_{\text {min }}$. But the galaxy-like object catalog is allowed to include objects with a lower $M_{\min }$, meaning that each "stellar" particle is considered a galaxy-like object. The influence of these two parameters on the galaxy mass function, $\eta$ and $M_{\min }$, are discussed in Appendix B.

Unless otherwise stated, the results of this paper are given for a $\Lambda$-cold dark matter model $(\Lambda-C D M)$. The parameters of the simulations are: $H_{0}=70 . \mathrm{km} \mathrm{s}^{-1} \mathrm{Mpc}^{-1}, \Omega_{\mathrm{K}}=0$, $\Omega_{\mathrm{m}}=0.3, \Omega_{\Lambda}=0.7, \Omega_{\mathrm{b}}=0.02 h^{-2}$ with $h=H_{0} / 100$. The initial density fluctuation spectrum uses the transfer functions taken from Bardeen et al. (1986) with a shape parameter given by Sugiyama (1995). The fluctuation spectrum is normalized to COBE data (Bunn \& White 1997) leading to a filtered dispersion at $R=8 h^{-1} \mathrm{Mpc}$ of $\sigma_{8}=0.91$. The number of dark 
Table 1. Parameters of the simulations. $L_{\mathrm{box}}$ is the comoving length of the computational volume, $\mathrm{d} x$ is the spatial resolution of the grid, $M_{\mathrm{dm}}$ is the mass of the dark matter particle and $M_{\mathrm{bm}}$ is the initial baryonic mass enclosed within a grid cell.

\begin{tabular}{lcccc}
\hline \hline Simulation & $\begin{array}{c}L_{\mathrm{box}} \\
\left(h^{-1} \mathrm{Mpc}\right)\end{array}$ & $\begin{array}{c}\mathrm{d} x \\
\left(h^{-1} \mathrm{kpc}\right)\end{array}$ & $\begin{array}{c}M_{\mathrm{dm}} \\
\left(M_{\odot}\right)\end{array}$ & $\begin{array}{c}M_{\mathrm{bm}} \\
\left(M_{\odot}\right)\end{array}$ \\
\hline & & & & \\
$G 0$ & 32. & 125. & $2.01 \times 10^{8}$ & $3.09 \times 10^{7}$ \\
$G 1, G N E$ & 16. & 62.5 & $2.51 \times 10^{7}$ & $3.87 \times 10^{6}$ \\
$G P$ & 11. & 43. & $8.17 \times 10^{6}$ & $1.25 \times 10^{6}$ \\
\hline
\end{tabular}

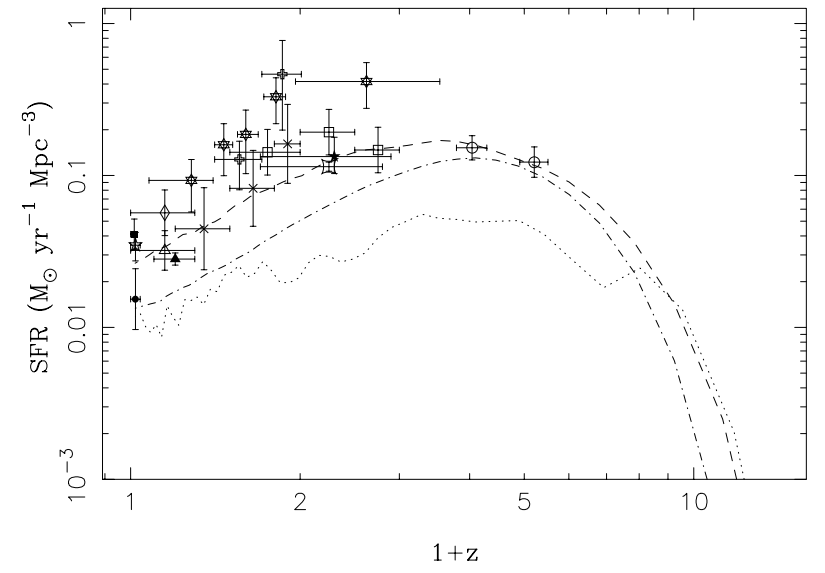

Fig. 3. Evolution with redshift of the cosmic "stellar" mass formation rate density for the $G 1$ (dashed line), $G 0$ (dot-dashed line) and $G P$ (dotted line) simulations. The observational data of the star formation rate density are overplotted for our cosmology: $\mathrm{H} \alpha$ data: Gallego et al. (1995) (filled circle), Tresse \& Maddox (1998) (filled triangle), Yan et al. (1999) (filled star), Hopkins et al. (2000) (hollow diamond); UV data: Connolly et al. (1997) (open square), Treyer et al. (1998) (open triangle), Steidel et al. (1999) (open circle), Sullivan et al. (2000) (diamond); FIR data: Rowan-Robinson et al. (1997) (heavy cross), Flores et al. (1999) (cross); $1.4 \mathrm{GHz}$ data: Condon (1989) (filled square), Haarsma et al. (2000) (six-pointed star), Serjeant et al. (2002) (open star). UV data and data by Gallego et al. (1995) are corrected for extinction.

matter particles is $N_{\mathrm{p}}=256^{3}$ and the number of grid cells is $N_{\mathrm{g}}=256^{3}$. Three computational volumes are used, described, as well as the simulation parameters, in Table 1 .

\section{Galaxy properties}

\subsection{Cosmic "stellar" mass formation rate density}

Figure 3 shows the redshift evolution of the "star" formation rate (SFR) density for the $G 0$ and $G 1$ simulations. We compute the amount of "stellar" mass formed per year and per unit of volume. The SFR density shows a strong decrease at low redshift and peaks around redshift $\sim 3$ in $G 0$ and 2.5 in $G 1$. However, the ratio between the SFR at the peak and the SFR at $z=6$ is lower than between the SFR at the peak and at $z=0, \operatorname{SFR}\left(z=z_{\text {peak }}\right) / S F R(z=6) \sim 3.2$ against $S F R\left(z=z_{\text {peak }}\right) / S F R(z=0) \sim 10$ in the $G 0$ simulation (these values become 2.8 and 6.3 in $G 1$, respectively). This trend is related to the hierarchical nature of cold dark matter models.

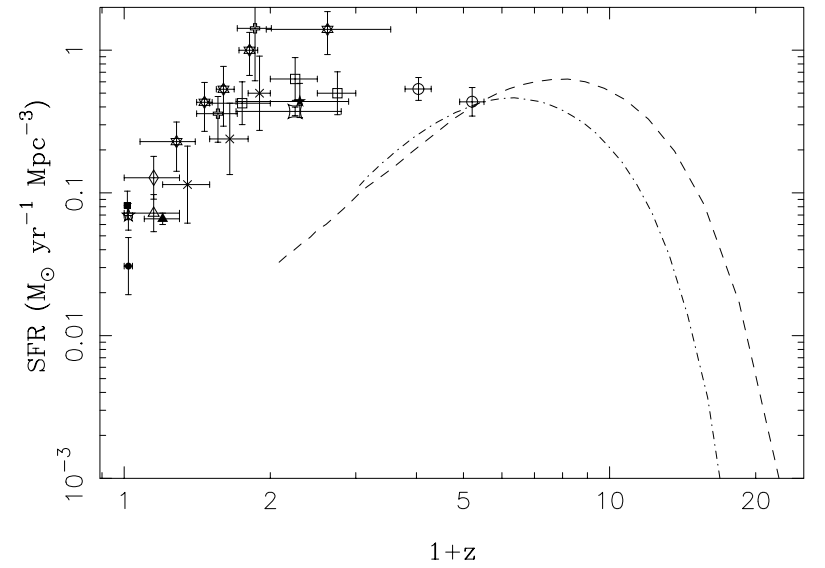

Fig. 4. Same as Fig. 3 but for a $S C D M$ scenario, for the $G 1$ (dashed line) and $G 0$ (dot-dashed line) simulations. The observation points are the same as described in Fig. 3 but overplotted for a SCDM cosmology.

At high redshift baryonic matter is accreted in dark matter potential wells and cools to form galaxy material. Then low-mass structures merge together to form larger mass units. Additional gas accreted is then shock heated towards higher temperatures leaving insufficient time for the gas to cool and condense. This results in the decrease in the star formation rate at low $z$.

Another illustration of the influence of the large scale structures on galaxy formation comes from the comparison with a different cosmological scenario. We run the same simulations but now computed for a standard cold dark matter model $(S C D M)$ with the following parameters: $H_{0}=$ $50 \mathrm{~km} \mathrm{~s}^{-1} \mathrm{Mpc}^{-1}, \Omega_{\mathrm{K}}=0 ., \Omega_{\mathrm{m}}=1 ., \Omega_{\Lambda}=0 ., \Omega_{\mathrm{b}}=0.02 h^{-2}$ with $h=H_{0} / 100$. As in the $\Lambda-C D M$ scenario, the fluctuation spectrum is normalized to COBE data, giving $\sigma_{8}=1.1$. For computational reasons the $S C D M G 1$ simulation is only performed up to $z=1$ and the $G 0$ up to $z=2$. The density fluctuation power spectrum has more power on small scales than the $\Lambda-C D M$ model, resulting in a larger amount of cold gas at high redshift. Differences between these two models are strikingly illustrated by the cosmic star formation rate density (Fig. 4). The higher amount of dark matter in the SCDM, resulting in a slightly larger $\sigma_{8}$ than in the $\Lambda-C D M$ scenario, creates deep potential wells at high redshift and as a result the peak of the SFR density is reached at $z=6$, or even higher $z$, leading to a steep decrease at low redshift.

Figure 3 shows that the SFR density depends on the spatial resolution of the simulations: the highest resolution simulation, $G 1$, has a SFR density amplitude higher at low redshift than the $G 0$ simulation. Decreasing the box length allows to include in the computational volume density fluctuations with lower wavelengths, resulting in a higher mass fraction of available cold gas (similar trends are discussed in Weinberg et al. 1999 and Ascasibar et al. 2002). Figures 5 and 6 display, for the same simulations as $G 0$ and $G 1$ but without galaxy formation, the evolution with redshift of the baryonic mass fraction in different ranges of temperature, corresponding to the main phases of the inter-galactic medium: the "diffuse" phase with $T<9 \times 10^{3} \mathrm{~K}$, the "cold" phase with temperature in 


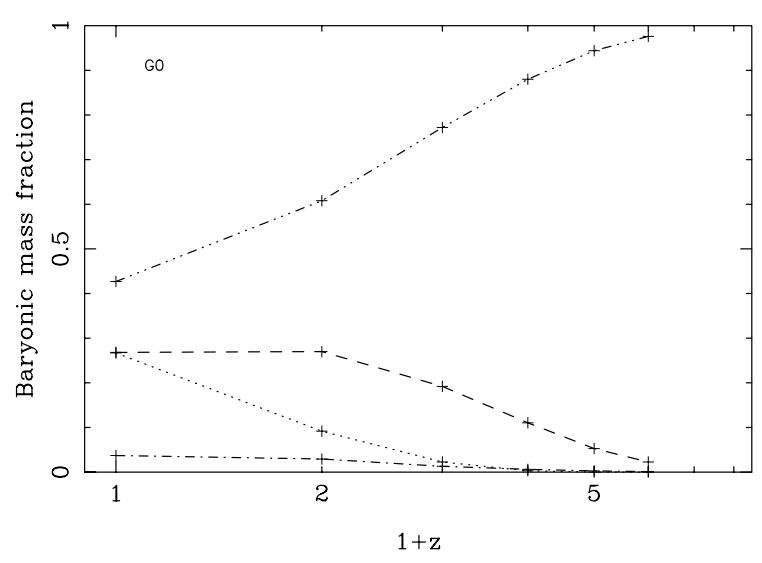

Fig. 5. Evolution with redshift of the baryonic mass fractions computed in different temperature ranges, for the $G 0$ simulation but without galaxy formation: $T<9 \times 10^{3} \mathrm{~K}$ ("diffuse", dot-dot-dashed line), $9 \times 10^{3} \leq T<2 \times 10^{4} \mathrm{~K}$ ("cold", dashed line), $2 \times 10^{4} \leq T<5 \times 10^{5} \mathrm{~K}$ ("warm", dot-dashed line), $T \geq 5 \times 10^{5} \mathrm{~K}$ ("hot", dotted line).

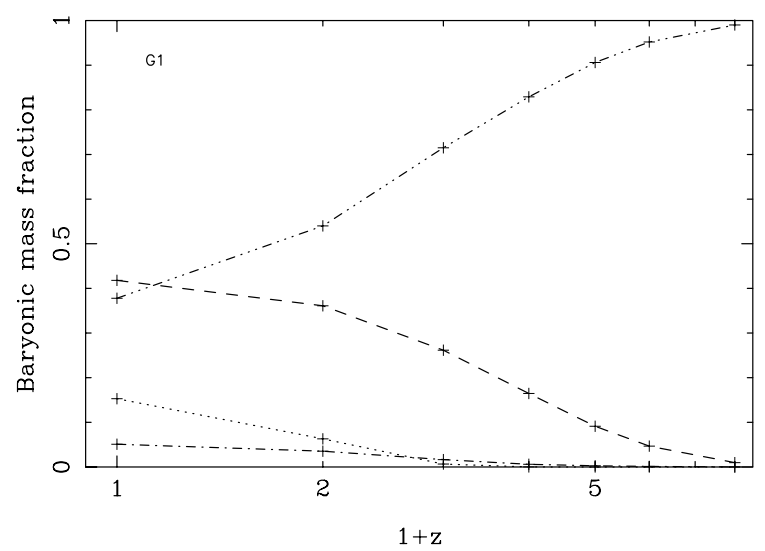

Fig. 6. Same as Fig. 5, but for the $G 1$ simulation without galaxy formation.

the range $9 \times 10^{3}-2 \times 10^{4} \mathrm{~K}$, the "warm" phase in the range $2 \times 10^{4}-5 \times 10^{5} \mathrm{~K}$ and the "hot" phase at $T \geq 5 \times 10^{5} \mathrm{~K}$. The increase in the amount of cold gas with decreasing redshift is less dramatic for the $G 0$ simulation: between $z=5$ and $z=0$, the cold gas mass fraction goes from $5.2 \%$ to $26 \%$ whereas it goes from $4.6 \%$ to $41 \%$ in $G 1$. However changing the resolution does not change the evolution of the SFR density and we are less interested in making quantitative estimates than analyzing qualitatively the process of galaxy formation.

Observational data of the star formation rate density are overplotted in Fig. 3. These data show a great disparity and the amplitude of the SFR density is expected to change due to different observational bias corrections (Hopkins et al. 2001). Nonetheless a strong decrease is observed at low redshift. Moreover observations do not currently agree whether the SFR density peaks after $z=1$ or reaches a plateau at higher redshift. Both star formation rate densities in our simulations reproduce the slope at low redshift and the amplitude obtained in the $G 1$ simulation is consistent with observations (contrary to the SCDM scenario showing a decrease at earlier epochs). This agreement and the previous discussion point out that galaxy formation is mainly driven by the amount of available cold gas
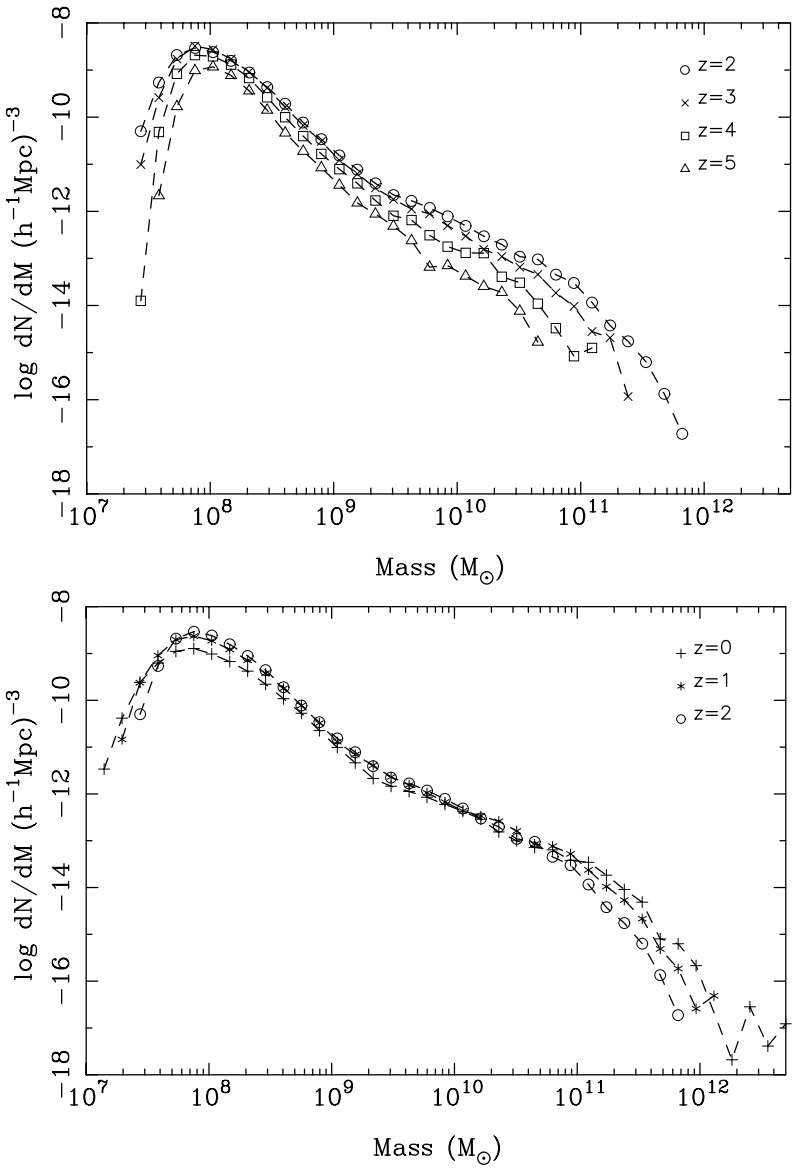

Fig. 7. Galaxy-like object mass function at different redshifts for the G0 simulation.

enclosed in gravitationally bound structures, the strongest constraint of the galaxy formation model lying in the condition $t_{\text {cool }}<t_{\text {ff }}$ (see also Pearce et al. 2001).

\subsection{Galaxy-like object mass function}

The rest of Sect. 3 is now devoted to the description of our two galaxy catalogs and examines an important property of galaxy formation, the galaxy-like object mass function. Discussions about their epoch of formation and their location inside dark matter halos will follow.

Figure 7 shows the cosmological evolution of the mass function of the galaxy-like objects in the $G 0$ simulation. The catalog covers a wide range a mass, from $10^{8} M_{\odot}$ up to $10^{12} M_{\odot}$. As we do not know if the decrease in the range $M<10^{8} M_{\odot}$ is a numerical effect (see also Murali et al. 2002), we discuss in the following the shape of the mass function for $M>10^{8} M_{\odot}$. At any redshift the comoving number density of objects per bin of mass increases as the mass decreases. This trend is different from the stellar particle mass functions (Fig. A.1). The galaxy mass function shows a clear evolution from $z=5$ to $z=0$ : more and more objects of high-mass are created, increasing the mass range of galaxies towards the highmass end. Since galaxy formation results from a hierarchical process, the mass of bigger objects increases with decreasing redshift, shifting the knee of the mass function towards larger 

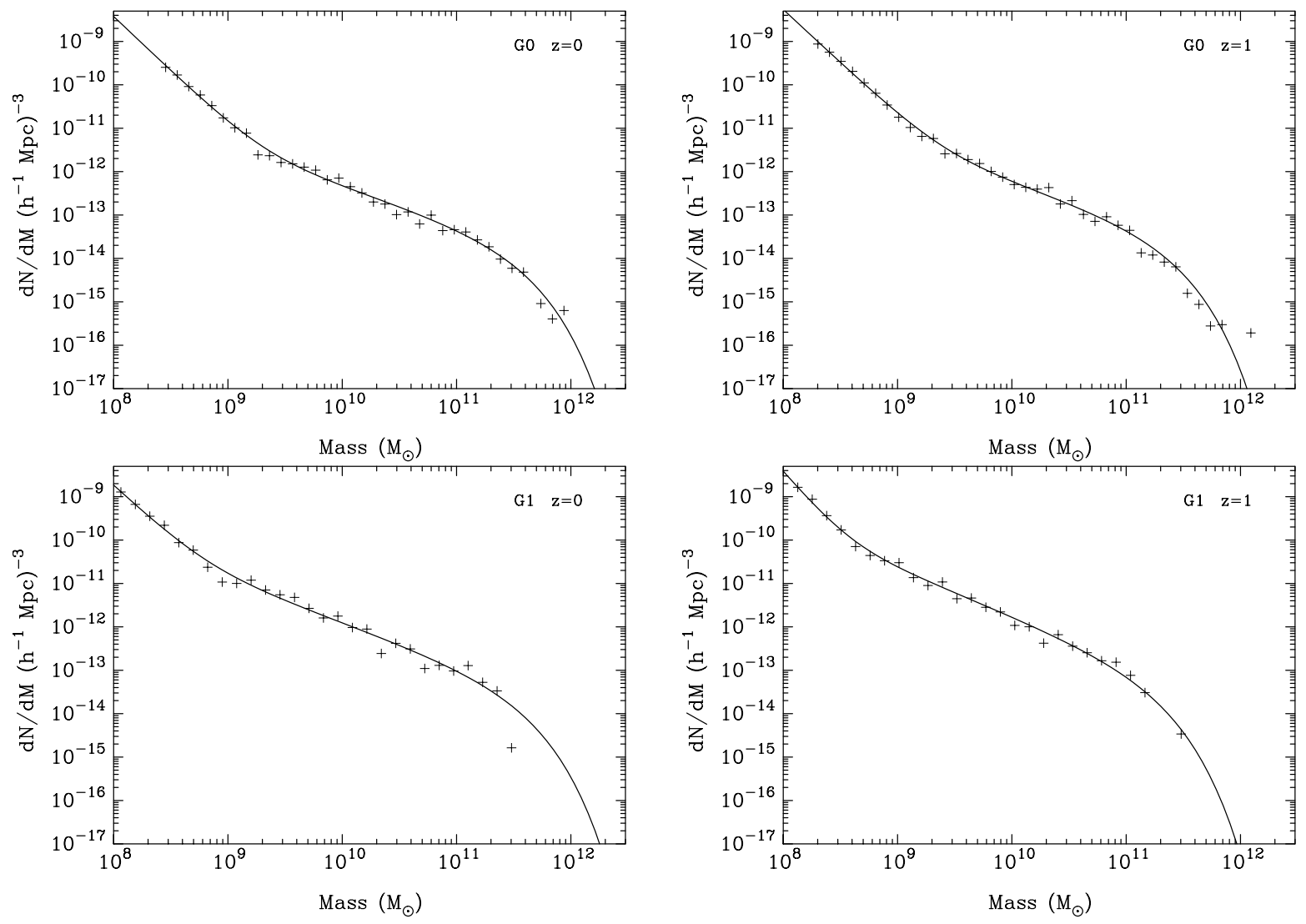

Fig. 8. Analytical fits (solid curve) of the galaxy-like object mass function (cross) for the $G 0$ and $G 1$ simulations at $z=0$ and $z=1$. Fits are computed using Eq. (11).

mass. On the other hand the low-mass end of the mass function shows an increase as redshift decreases to only $z=2$. At lower redshift the trend is inverted with a decrease in the number density per bin of mass of low-mass objects.

On the whole the galaxy mass function shows a characteristic shape: a strong decrease at the high-mass end beyond a characteristic mass, preceded by a shallower slope in the intermediate mass range, between $10^{9}-10^{11} M_{\odot}$, and a steeper slope at the low-mass end up to $10^{8} M_{\odot}$. Note the sharp transition at $z=0$ around a few times $10^{9} M_{\odot}$, shown more clearly in Fig. 8 .

Figure 7 presents one of the main results of the paper: the galaxy mass function is significantly dominated by a low-mass galaxy population, $M<10^{10} M_{\odot}$, whatever the redshift is. This population can be linked to the observed faint luminosity galaxy population (Loveday 1998; Norberg et al. 2002, and references therein). In fact this faint population covers a wide variety of galaxies: galaxies with or without emission lines (Zucca et al. 1997), galaxies characterized by a significant star formation activity (Lin et al. 1996; Loveday et al. 1999), dwarf galaxies of morphological and spectral late types (Marzke et al. 1994, 1998), low surface brightness galaxies (Sprayberry et al. 1997), blue compact objects (Guzman et al. 1997). The discussion below about the epoch of formation of the galaxy-like objects will show that, in the simulations also, the low-mass end of the galaxy mass function includes an inhomogeneous population. This population of faint galaxies is important for galaxy evolution, since by their number they are likely to contribute to the cosmic star formation rate density. Their relationship to the environment and their implication in galaxy mergers still needs to be addressed.

The galaxy luminosity function is generally fitted by a standard Schechter function combining a power-law and an exponential function at the bright end (Schechter 1976):

$\phi(L)=\frac{\phi_{*}}{L_{*}}\left(\frac{L}{L_{*}}\right)^{\alpha} \exp \left(-\frac{L}{L_{*}}\right)$

where $\alpha$ is the slope of the power-law, $L_{*}$ is the characteristic luminosity at the break, and $\phi_{*}$ is a normalization parameter. Table 2 lists parameter fits, extracted from the literature, of the galaxy luminosity function for different surveys. In order to differentiate mass from magnitude, the symbol $\mathcal{M}$ is used for magnitude in this table and throughout the paper. As pointed out in the introduction, discrepancies remain between surveys, enlightening the fact these surveys cover different galaxy populations. Observational results show that the galaxy luminosity function is different for galaxy populations selected by color, morphology or environnement. The $2 d \mathrm{~F}$ survey, for instance, shows how the shape of the galaxy luminosity function changes when galaxies are divided according to their star formation activity (Folkes et al. 1999).

Most of these surveys use a single power-law Schechter function and the $\alpha$ slope is generally found to be in the range -0.9 and -1.2 . A number of estimates of the galaxy luminosity function conclude that it cannot be described correctly, in the 
Table 2. Fit parameters of the galaxy luminosity function extracted from the literature for different surveys using a standard Schechter function (Eq. (10)). Magnitudes are computed for $h=0.7$. The normalization parameter is expressed in $h^{3} \mathrm{Mpc}^{-3}$. A two power-law Schechter function is used in Loveday (1997) with a correction for the $\beta$ parameter in Loveday (1998). Zucca et al. (1997) describes the faint part of the galaxy luminosity function by a power-law introducing the $\beta$ slope and the magnitude $\mathcal{M}_{\mathrm{c}}$ (line noted $(b)$ ).

\begin{tabular}{llll}
\hline \hline Reference & $\phi^{*}$ & $\alpha$ & $\mathcal{M}_{*}$ \\
\hline B band & & & \\
Loveday et al. (1992) & 0.014 & -0.97 & -20.27 \\
Marzke et al. (1994) & 0.04 & -1.0 & -20.02 \\
Ellis et al. (1996) & & & \\
Loveday (1997) & 0.0154 & -0.94 & -20.42 \\
& & $-1.82(\beta)$ & $-14.84\left(\mathcal{M}_{\mathrm{t}}\right)$ \\
Zucca et al. (1997) & 0.02 & -1.22 & -20.38 \\
Zucca et al. (1997) (b) & 0.021 & -1.16 & -20.34 \\
& & $-1.57(\beta)$ & $-17.76\left(\mathcal{M}_{\mathrm{c}}\right)$ \\
Ratcliffe et al. (1998) & 0.017 & -1.04 & -20.45 \\
Norberg et al. (2002) & 0.0168 & -1.21 & -20.43 \\
& & & \\
$K$ band & & & \\
Glazebrook et al. (1995) & 0.029 & -1.04 & -23.5 \\
Gardner et al. (1997) & 0.0166 & -0.91 & -23.9 \\
Loveday (2000) & 0.012 & -1.16 & -24.35 \\
Kochanek et al. (2001) & 0.0116 & -1.09 & -24.16 \\
Cole et al. (2001) & 0.0116 & -0.93 & -24.13 \\
\hline
\end{tabular}

entire range of luminosity and specially at $\mathcal{M}_{B}>-15$, by a single power-law Schechter function (Loveday 1998). Two works, reported in Table 2, use a non-standard Schechter function to parameterize the galaxy luminosity function: a two power-law Schechter function described below, is used in Loveday (1997) and two analytical functions are used in Zucca et al. (1997), a standard Schechter function and a power-law at the faint luminosity end. Both report slopes in the faint luminosity range of -1.82 and -1.57 , respectively.

The mass function of the galaxy-like objects in Fig. 7 clearly suggests similar conclusion. Therefore to account for the low-mass galaxy population we choose a two power-law Schechter function now combining a standard Schechter function with a $\beta$ power-law at the low-mass end (Loveday 1997):

$\frac{\mathrm{d} N}{\mathrm{~d} M}=\frac{\phi_{*}}{M_{*}}\left(\frac{M}{M_{*}}\right)^{\alpha} \exp \left(-\frac{M}{M_{*}}\right)\left[1+\left(\frac{M}{M_{\mathrm{t}}}\right)^{\beta}\right]$

where $\phi(M)=\mathrm{d} N / \mathrm{d} M$ is the numerical density of objects per interval of mass and per unit of volume, $\phi^{*}, M_{*}$ and $\alpha$ are analogous parameters as used in Eq. (10), and $M_{\mathrm{t}}$ is the transitional mass between the two power-laws. The mass function of the galaxy-like objects is fitted, in the range $M>10^{8} M_{\odot}$, using a least square method weighted by the mass function itself to ensure the statistical reliability of the result. Figure 8 and Table 3 give fits and parameters determined at $z=0$ and $z=1$ for the $G 0$ and $G 1$ simulations. At $z=0$ the characteristic mass at the high-mass end is roughly similar for the two resolutions, around $2 \times 10^{11} M_{\odot}$. The $\alpha$ slope is quite shallow, around or higher than -1 , whereas the $\beta$ slope is less than -1.5 . These
Table 3. Fit parameters (Eq. (11)) computed for the galaxy-like object mass function (Fig. 8) for the $G 0$ and $G 1$ simulations at $z=0$ and $z=1$. The characteristic masses are expressed in $M_{\odot}$ and the normalization parameter in $h^{3} \mathrm{Mpc}^{-3}$.

\begin{tabular}{cccccc}
\hline \hline \multicolumn{1}{c}{$\phi_{*}$} & $M_{*}$ & $\alpha$ & $M_{\mathrm{t}}$ & $\beta$ \\
\hline \multirow{2}{*}{$G 0$} & & & & & \\
$z=0$ & 0.00720 & $2.53 \times 10^{11}$ & -0.86 & $2.13 \times 10^{9}$ & -1.65 \\
$z=1$ & 0.00824 & $1.67 \times 10^{11}$ & -0.87 & $2.55 \times 10^{9}$ & -1.59 \\
& & & & & \\
$G 1$ & & & & & \\
$z=0$ & 0.0144 & $2.64 \times 10^{11}$ & -0.96 & $6.35 \times 10^{8}$ & -1.52 \\
$z=1$ & 0.0146 & $1.29 \times 10^{11}$ & -1.08 & $3.69 \times 10^{8}$ & -2.02 \\
\hline
\end{tabular}

values are consistent with the ones obtained in different surveys (Table 2). It is quite remarkable that such a simple galaxy formation model reproduces the shape of the galaxy luminosity function. The $\alpha$ slope does not show strong evolution between $z=0$ and $z=1$, contrary to the characteristic mass decreasing as the redshift increases. The transitional mass and the normalization parameter show clearly the influence of resolution: the $\alpha$ power-law extends on a higher mass range for the highest resolution, changing the transitional mass: $M_{\mathrm{t}} \sim 6 \times 10^{8} M_{\odot}$ for the $G 1$ simulation whereas $M_{\mathrm{t}} \sim 2 \times 10^{9} M_{\odot}$ in $G 0$. The bottom panel in Fig. A.2 shows a comparison between both resolutions at $z=0$. Indeed a higher resolution allows the formation of lower mass galaxy-like objects. The mass functions tend to be steeper in the intermediate range at $z=1$, and the characteristic mass $M_{*}$ decreases. We will return to these differences at the end of this section.

The integration of the galaxy mass function gives the mean mass density, $j_{\mathrm{M}}=\int \phi(M) M \mathrm{~d} M$. Using the fits displayed in Table 3 we compute $j_{M}$ over the range $M>10^{8} M_{\odot}$. At $z=0$ this quantity is $8.73 \times 10^{8}$ and $1.84 \times 10^{9} h M_{\odot} \mathrm{Mpc}^{-3}$ for the $G 0$ and $G 1$ simulations, respectively. At $z=1$ the mean mass densities are lower: $6.9 \times 10^{8}$ and $9.9 \times 10^{8} h M_{\odot} \mathrm{Mpc}^{-3}$ for the same simulations, respectively. Normalized to the critical density parameter at $z=0, \rho_{c, 0}=2.67 \times 10^{11} h^{2} M_{\odot} \mathrm{Mpc}^{-3}$, the stellar density parameter decreases from $\Omega_{*}=0.00451$ to 0.00357 between $z=0$ and $z=1$ for the $G 0$ simulation, and from $\Omega_{*}=0.00954$ to 0.00511 for $G 1$. The values at $z=0$ can be compared to Fukugita et al. (1998), reporting a central value of $\Omega_{*}=0.0035$ (see their Table 3 ) which accounts for stars in spheroids, disks and irregulars.

In order to compare the characteristic masses $M_{*}$ and $M_{\mathrm{t}}$ with the characteristic magnitudes of the galaxy luminosity function, we use the mean mass densities to derive the massto-light ratios of the galaxy-like object catalogs. We express the mass-to-light ratio as $\gamma=j_{\mathrm{M}} / j_{\mathrm{L}}$ where $j_{\mathrm{L}}$ is the mean luminosity density, $j_{\mathrm{L}}=\int_{0}^{\infty} \phi(L) L \mathrm{~d} L$. Note that this ratio is a "stellar" mass-to-light ratio since the galaxy-like objects enclose only "stellar" material. The mean luminosity density is taken to be $2.5 \times 10^{8}$ and $5 \times 10^{8} h L_{\odot} \mathrm{Mpc}^{-3}$ in the $b_{\mathrm{J}}$ and $K$ bands, respectively (Cole et al. 2001). Table 4 displays the mass-to-light ratios in each band and for the two resolutions. Values are higher in the $B$ band and also for the highest resolution simulation. They are between 1.7 and 7.4, consistent with 
Table 4. Mass-luminosity ratios and characteristic magnitudes in the $B$ and $K$ bands for the $G 0$ and $G 1$ simulations at $z=0$.

\begin{tabular}{ccccc}
\hline \hline Simulation & $\gamma$ & $\mathcal{M}_{*}$ & $\mathcal{M}_{\mathrm{t}}$ & \\
\hline & & & & \\
$G 0$ & 3.5 & -21.8 & -16.6 & $B$ band \\
$G 0$ & 1.7 & -24.6 & -19.4 & $K$ band \\
& & & & \\
$G 1$ & 7.4 & -21.1 & -14.5 & $B$ band \\
$G 1$ & 3.7 & -23.8 & -17.3 & $K$ band \\
\hline
\end{tabular}

Table 5. Characteristic magnitudes in the $B$ and $K$ bands for the $G 0$ and $G 1$ simulations at $z=1$ estimated with the mass-to-light ratios at $z=0$ given in Table 4 .

\begin{tabular}{lccc}
\hline \hline Simulation & $\mathcal{M}_{*}$ & $\mathcal{M}_{\mathrm{t}}$ & \\
\hline$G 0(z=1)$ & -21.4 & -16.8 & $B$ band \\
$G 0(z=1)$ & -24.2 & -19.6 & $K$ band \\
& & & \\
$G 1(z=1)$ & -20.3 & -13.9 & $B$ band \\
$G 1(z=1)$ & -23.0 & -16.7 & $K$ band \\
\hline
\end{tabular}

observational results (Fukugita et al. 1998, give mass-to-light ratios of 6.5 for spheroids and 1.5 for disks). Recall that these mass-to-light ratios are computed over an extensive range of mass, and that this quantity is likely to depend on mass.

From these mass-to-light ratios we estimate the characteristic magnitudes $\mathcal{M}^{*}$ and $\mathcal{M}_{\mathrm{t}}$ corresponding to the characteristic masses $M_{*}$ and $M_{t}$ (in the expression $\mathcal{M}-\mathcal{M}_{\odot}=-2.5 \times$ $\log \left(L / L_{\odot}\right)$ solar magnitude values are taken to be $\mathcal{M}_{b_{\mathrm{J}}}^{\odot}=5.3$ and $\mathcal{M}_{K}^{\odot}=3.3$ ). Results are given in Table 4 . In the $B$ band $\mathcal{M}_{*}$ is brighter than observational values (between -20 and -20.5 , see Table 2). In the $K$ band $\mathcal{M}_{*}$ is in agreement with observations (around -24). Indeed the infrared band is a better tracer of the stellar content, and is not dominated by young stellar population strongly emitting in ultraviolet and affected by dust extinction. Converting mass into luminosity using mass-to-light ratios is then expected to be more reliable in the $K$ band. The transitional magnitudes defined in Loveday (1997) and Zucca et al. (1997) are -14.8 and -17.7 (Table 2), respectively, and our transitional magnitudes in Table 4 are consistent with these values. Making the crude assumption that the mass-to-light ratio is constant between $z=0$ and $z=1$, we derive the characteristic magnitudes at $z=1$ corresponding to the characteristic masses quoted in Table 3 . Table 5 shows that $\mathcal{M}_{*}$ is roughly half a magnitude fainter in the $B$ band than at $z=0$ and this trend is similar to the evolution with redshift of $L_{*}$ in the survey Autofib (Ellis et al. 1996).

Before going further into the description of the galaxy population we compare the galaxy mass function with the dark matter halo mass function. Figure 9 shows that the mass range extends from $10^{9}$ to more than $10^{14} M_{\odot}$. At any redshift the number density of dark matter halos per bin of mass increases as the mass decreases. No strong decrease at the high-mass end is seen, as in the galaxy mass function, at low redshift because of our small computational volume, this behavior being expected at much higher mass (Jenkins et al. 2001). A slight

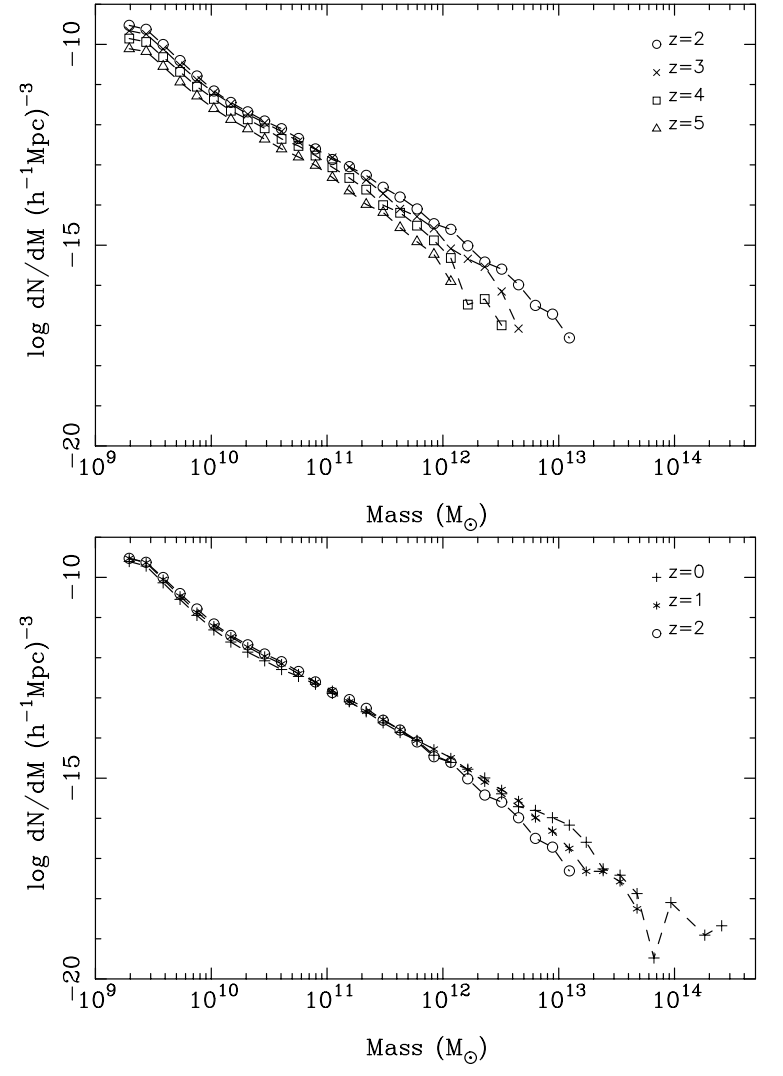

Fig. 9. Dark matter halo mass function at different redshifts for the $G 0$ simulation.

decrease is seen around $10^{13} M_{\odot}$ and we thus adopt a two power-law fit in the range $M>10^{9} M_{\odot}$, such as:

$\frac{\mathrm{d} N}{\mathrm{~d} M}=\frac{\phi_{*}}{M_{\mathrm{t}_{1}}}\left(\frac{M}{M_{\mathrm{t}_{1}}}\right)^{\alpha}\left[1+\left(\frac{M}{M_{\mathrm{t}_{1}}}\right)^{\gamma}\right]^{-1}$

where $\phi^{*}$ the normalization parameter, $\alpha$ and $\gamma$ the slopes in the high and low-mass range, respectively, and $M_{\mathrm{t}_{1}}$ a transitional mass between the two power-laws. Fits and their parameters at $z=1$ and $z=0$ are described in Fig. 10 (solid curve) and Table 6. The $M_{\mathrm{t}_{1}}$ value is $3.4 \times 10^{13} M_{\odot}$ at $z=0$ and around $9 \times 10^{12}$ at $z=1$. The halo mass functions are much steeper than the galaxy mass functions in the intermediate mass range.

However the halo mass functions steepens at the low-mass end, $M<10^{10} M_{\odot}$, and the analytical function in Eq. (12) does not correctly fit the mass functions for the entire mass range. We then use a three power-law function by multiplying $\mathrm{d} N / \mathrm{d} M$ by the term $\left[1+\left(M / M_{\mathrm{t}}\right)^{\beta}\right]$, similarly to the galaxy mass function. The fit is shown in Fig. 10 by the dashed curve and Table 6 gives this second set of now 6 parameters.

\subsection{Epoch of formation}

The galaxy mass function characterizes the galaxy population at a given epoch and does not give any information about the background of objects. Catalogs are likely to mix galaxies with different properties. The epoch of formation is then a first insight into their history. Since each stellar particle carries a formation epoch $a_{*}$ and a mass $m_{*}$, the epoch of formation of any 
Table 6. Fit parameters (Eq. (12)) computed for the dark matter halo mass function (Fig. 10) for the $G 0$ simulation at $z=0$ and $z=1$. The characteristic masses are expressed in $M_{\odot}$ and the normalization parameter in $h^{3} \mathrm{Mpc}^{-3}$.

\begin{tabular}{lllllll}
\hline \hline & $\phi_{*}$ & $M_{\mathrm{t}_{1}}$ & $\alpha$ & $\gamma$ & $M_{\mathrm{t}}$ & $\beta$ \\
\hline \multirow{2}{*}{$z=0$} & 0.000426 & $3.40 \times 10^{13}$ & -1.60 & 1.39 & & \\
& 0.000743 & $2.95 \times 10^{13}$ & -1.50 & 1.40 & $7.13 \times 10^{9}$ & -1.86 \\
$z=1$ & 0.001 & $9.02 \times 10^{12}$ & -1.62 & 1.57 & & \\
& 0.001 & $9.02 \times 10^{12}$ & -1.60 & 1.57 & $6.11 \times 10^{9}$ & -1.89 \\
\hline
\end{tabular}
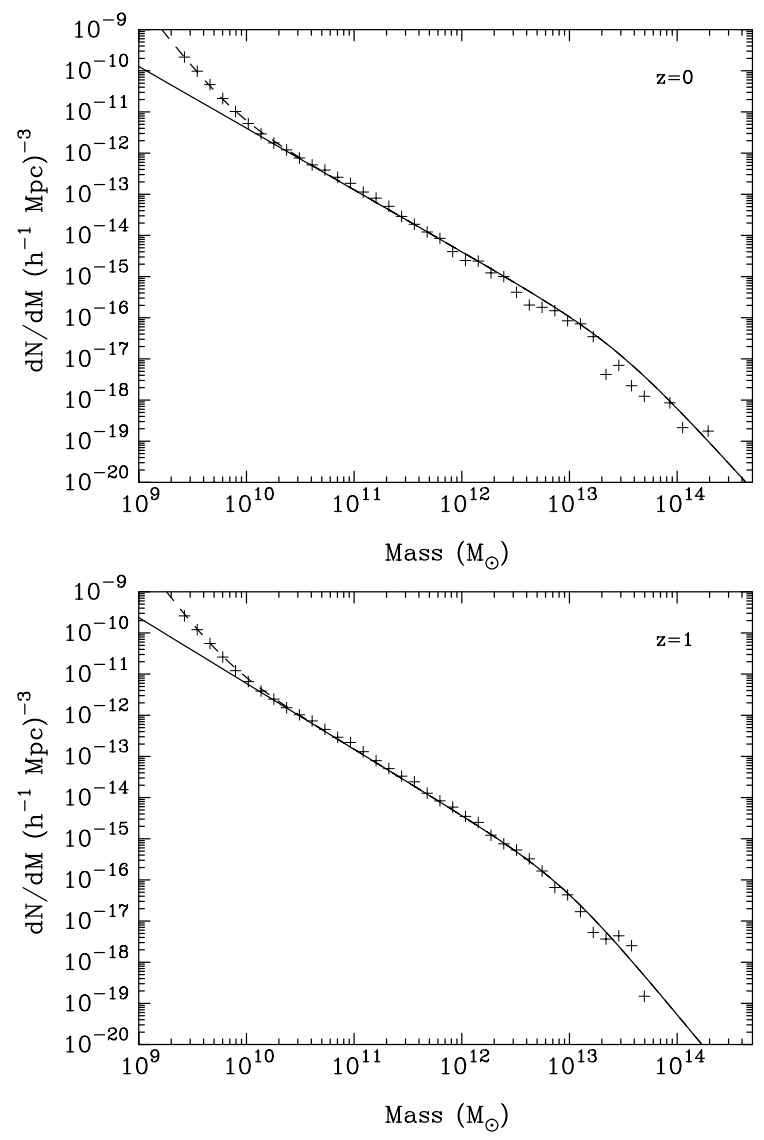

Fig. 10. Analytical fits (solid curve) of the dark matter halo mass function (cross) for the $G 0$ simulation at $z=0$ and $z=1$, computed using Eq. (12). Dashed curve (identical to the solid curve at $M>10^{10} M_{\odot}$ ) is a three power-law analytical fit.

object is determined from each formation epoch of its stellar particles weighted by their mass, $\sum\left(a_{*} m_{*}\right) / \sum m_{*}$.

Figure 11 displays the conditional mean of the formation epoch of galaxies given a mass $M,\langle t \mid M\rangle$ (the age is the Hubble time minus the epoch of formation), computed for the cata$\log$ at $z=0$. The dispersion around the mean is shown by the error bars. This quantity is only shown for the $G 0$ simulation as the conclusions are the same for $G 1$. The epoch of formation decreases with increasing mass, for $M>10^{11} M_{\odot}$ galaxies, whereas the low-mass galaxies have an epoch of formation globally constant. High-mass objects show early epoch of formations (see also Pearce et al. 2001) but low-mass objects have formed more recently. The low dispersion around the conditional mean for the high-mass galaxies reflect the fact that their star formation has considerably slowed down at low

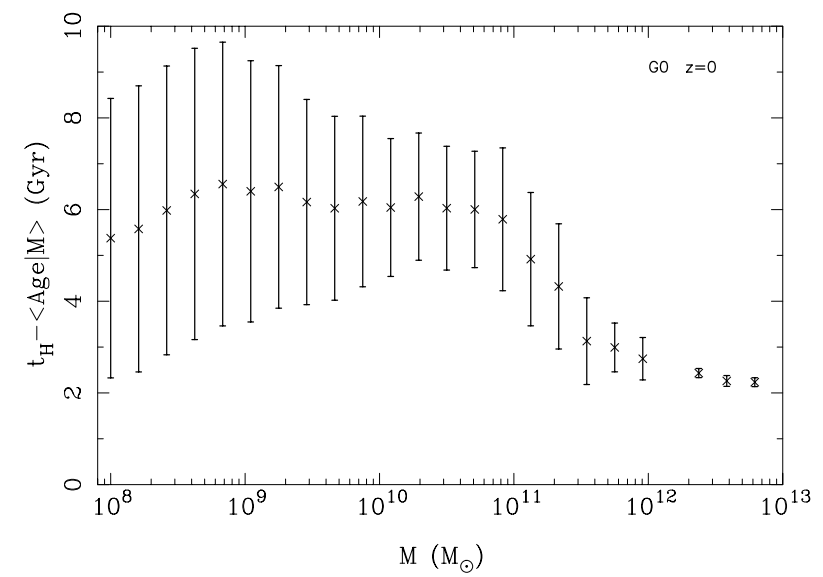

Fig. 11. Conditional mean of the galaxy-like object epoch of formation given a mass $M,\langle t \mid M\rangle$, for the $G 0$ simulation catalog at $z=0$ (today is at the upper end of the ordinate axis). The dispersion $\pm \sigma$ around the conditional mean is shown by the error bars.

redshift, contrary to the low-mass galaxies showing a large dispersion. This suggests that a part of the low-mass objects underwent very recently, or currently have, a star formation activity at low redshift. Another part of these objects have formed, like the high-mass objects, at high redshift and have stopped their star formation. Our results show that most of the stellar mass have formed by redshift $z=1,68 \%$ in the $G 0$ simulation $(37 \%$ by redshift $z=2$ and $16 \%$ by redshift $z=3$ ). In the $G 1$ simulation $61 \%$ have formed by redshift $z=1$ and $12 \%$ by redshift $z=3$. This trend and the fact that the most massive systems have quite old epochs of formation are similar a part of Springel \& Hernquist (2003) conclusions, although they have conducted a sophisticated study of the evolution of the star formation rate, using a large set of high-resolution simulations based on SPH methods and including star formation, supernova feedback and galactic outflows. Therefore the galaxy catalog seems to be populated on the one hand by high-mass galaxies being in majority early-formed galaxies (see Figs. 8 and 11). On the other hand, the low-mass end of the galaxy mass function is dominated either by early-formed galaxies or late-formed galaxies with a star formation activity. Separating these two last sub-populations would change the shape of the galaxy mass function. Regarding the fact that the faint end of the galaxy luminosity function is likely to be populated by starforming galaxies (Zucca et al. 1997; Madgwick 2002), this numerical result is quite encouraging.

The population of high-mass, early-formed galaxies could be the observational counterpart of red, passive elliptical 

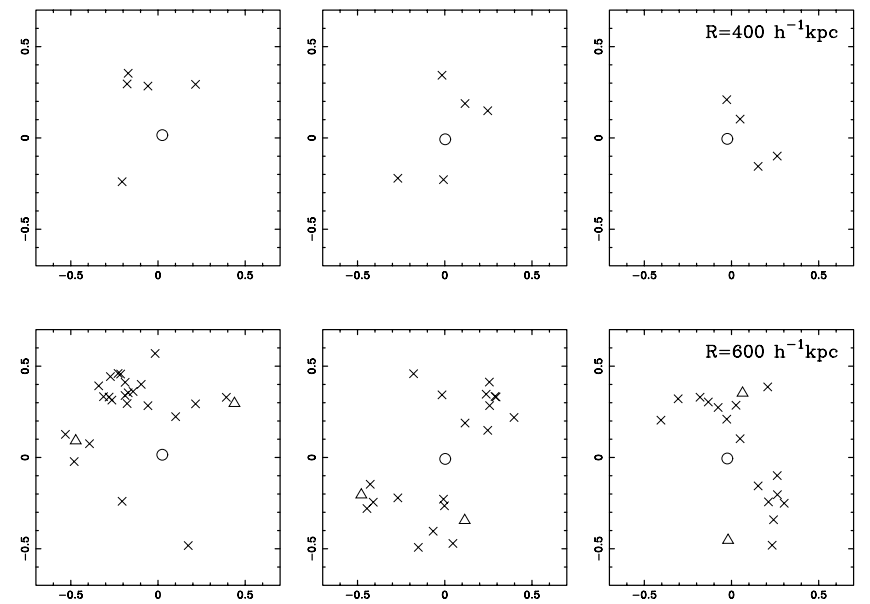

Fig. 12. Top panel: projected distribution of the galaxy-like objects inside a radius $<R=400 h^{-1} \mathrm{kpc}$ around the mass center of three dark matter halos with a mass higher than $10^{13} M_{\odot}$, for the $G 0$ simulation. The halo mass centers are at the origin of each plots, scaled in $h^{-1}$ Mpc. Each symbol represents the mass range of galaxies: $10^{8}<$ $M<10^{9} M_{\odot}$ (cross), $10^{9}<M<10^{11} M_{\odot}$ (triangle), $M>10^{11} M_{\odot}$ (circle). Lower panel: same as the top panel, but for $R=600 \mathrm{~h}^{-1} \mathrm{kpc}$.

galaxies in the center of galaxy clusters. To address this issue we focus on the galaxy-like object distribution inside the highest mass dark matter halos, with $M>10^{13} M_{\odot}$. Figure 12 displays the galaxy distribution at a distance $<R$ around the mass center of three dark matter halos, randomly chosen in the computational volume among the most massive ones. Galaxies are plotted, with symbols according to their mass range, inside a radius of $R=400$ (top panel) or $600 \mathrm{~h}^{-1} \mathrm{kpc}$ (lower panel) around each mass center halos. Dark matter halos host a whole population of galaxies, from $M=10^{8}$ to more than $10^{11} M_{\odot}$.

It is remarkable that each high-mass halo includes in its center a high-mass galaxy-like object (whose position at center is not defined a priori). Moreover the biggest halo contains the biggest galaxy-like object of the catalog. It has been pointed out that the most massive galaxy-like objects are also old objects and this result is consistent with the observational evidence that galaxy clusters have $\mathrm{cD}$ type galaxy in their center. It is interesting to note that inside a radius of $R=400 \mathrm{~h}^{-1} \mathrm{kpc}$ no galaxylike object with intermediate mass, between $10^{9}-10^{11} M_{\odot}$, is found in the proximity of the high-mass galaxy, although lowmass objects, $M<10^{9} M_{\odot}$, are present. Galaxies of intermediate mass appear when the radius around the mass center increases (lower panel).

\section{Photoionization processes}

We now turn to the $G P$ simulation including an ultraviolet background radiation (see Sect. 2) to analyze the influence of photoionization processes on the galaxy-like object properties. The same properties as in the previous section are discussed. Figure 3 overplots the cosmic star formation rate density for the $G P$ simulation. Although the amplitude is lower than for $G 1$, the general trend is not affected by photoionization processes and the SFR density decreases from $z \sim 2$ to the present. Recall that the $G P$ simulation has a smaller computational box length

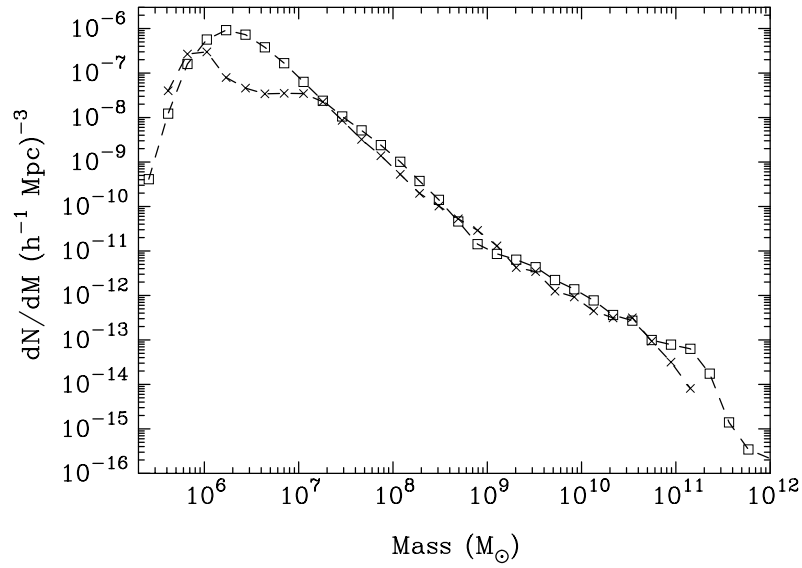

Fig. 13. Galaxy-like object mass function for the $G P$ (cross) and $G 1$ (square) simulations at $z=0$.

than $G 0$ and $G 1$ and that non-linear long wavelengths are likely to be missing at low redshift. A dip appears around $z=6$ (Barkana \& Loeb 2000) suggesting that low-mass stellar particles do not form above this redshift in low-mass structures where gas is now heated by photoionization processes. Indeed the gas distribution in the temperature-density diagram (middle panel in Fig. 2) shows that these processes are dominant over cooling processes only in low and middle density regions.

Figure 13 compares the galaxy mass function for the $G P$ and $G 1$ simulations at $z=0$. It reveals the influence of photoionization in low-density regions: the most dramatic differences are seen at the low-mass end, $M<10^{7} M_{\odot}$. The slopes and characteristic masses are similar as for $G 1$. The slight decrease at the high-mass end is likely due to the smaller computational volume. The formation epochs of galaxies are plotted in Fig. 14 down to $10^{6} M_{\odot}$, below this mass the galaxy-mass function decreases. The plot presents much more dispersion at $M>10^{8} M_{\odot}$ than in Fig. 11, the conditional mean formation epoch varying between 4 and 9 Gyr instead around 6 in the G0 simulation. Galaxies with a mass lower than $10^{8} M_{\odot}$ form at early epochs since the formation of low-mass stellar particle stops at low redshift (see Fig. A.1). Similarly to previous results (see Fig. 11) the highest mass galaxies seem to form at early epochs. Our results suggest that, with our choice for the $F(z)$ function, the photoionization processes have no dramatic influence on galaxy formation over a mass range higher than $10^{7} M_{\odot}$ (see also Quinn et al. 1996; Weinberg et al. 1997). More numerical investigations are nevertheless required to allow definitive conclusions.

\section{Non-equipartition processes}

We have investigated in Paper I the influence of additional dissipative processes on the cosmological plasma: nonequipartition processes between ions, neutrals and electrons may change the gas thermodynamic properties. The astrophysical implications are now discussed and quantified.

Paper I shows that the low-density, outer regions of gravitationally bound structures are found to be warmer in simulations including non-equipartition processes than in simulations 


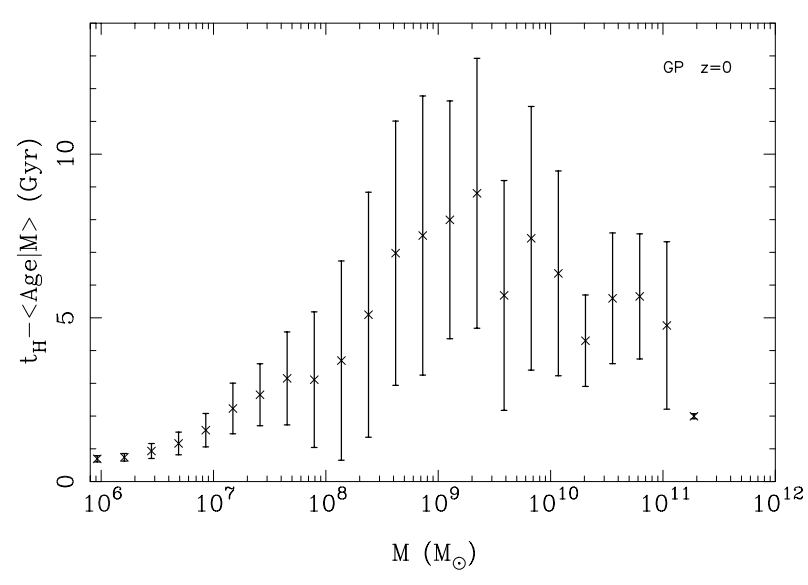

Fig. 14. Same as Fig. 11 but for the GP simulation.

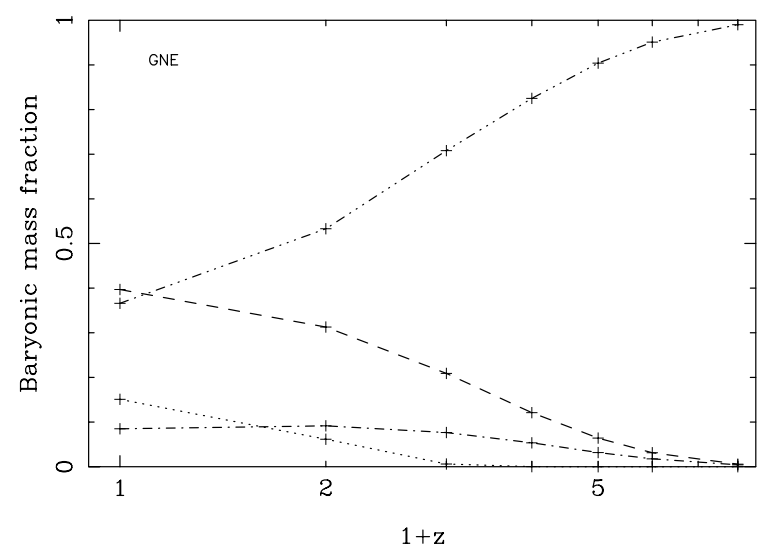

Fig. 15. Same as Fig. 5, but for the GNE simulation without galaxy formation.

in which equipartition is forced. This results in a warm gas fraction, illustrated by the gas distribution in Fig. 2 (bottom panel). Moreover we show that the cooling timescale of the warm plasma is longer than the cooling timescale of the same regions in simulations with forced equipartition. Figure 15 gives the baryonic mass fractions in different ranges of temperature for the simulation including non-equipartition processes from Paper I, the GNE simulation but without galaxy formation, and should be compared with Fig. 6 for $G 1$ : the fraction of gas with a temperature higher than $9 \times 10^{3} \mathrm{~K}$ is similar in both figures but the gas is not distributed in the same phases: at $z=5$, for example, $3.1 \%$ of the plasma constitutes the warm phase and $6.4 \%$ the cold phase in the simulation with non-equipartition processes, whereas these phases are $0.2 \%$ and $9.1 \%$ in $G 1$, respectively. The decrease in available cold gas needed for a galaxy to form is expected to affect the galaxy formation process.

We then analyze the GNE simulation, the same simulation as performed in Paper I but now including galaxy formation (Table 1). As the influence of non-equipartition processes is likely to be dominant at epochs before the end of the reionization epoch, we do not include photoionization processes and discuss the results at redshifts lower than 10 to mimic what happened before this epoch.

Figure 16 compares the evolution with redshift of the stellar particle mean mass between the GNE and $G 1$ simulations. The

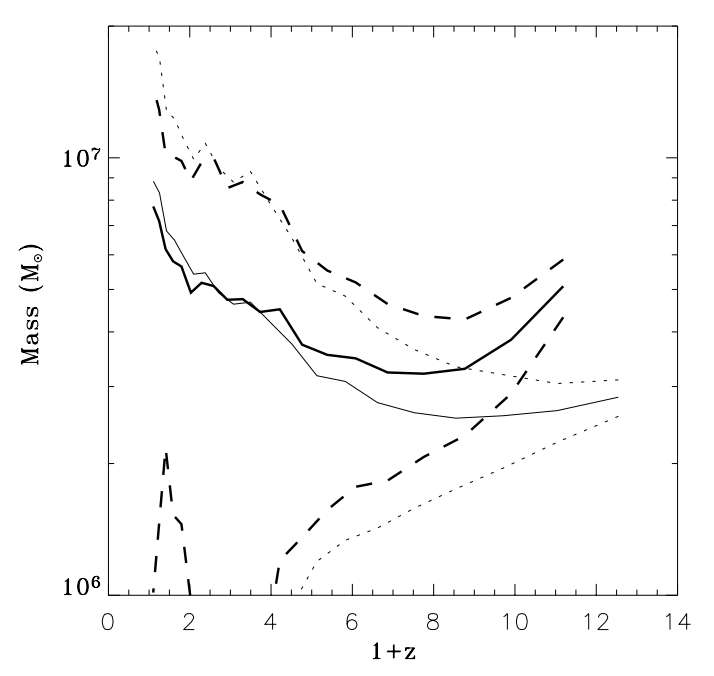

Fig. 16. Evolution with redshift of the stellar particle mean mass (solid lines) with the dispersion $\pm \sigma$ around the mean (dashed and dotted lines) for the $G N E$ (thick lines) and $G 1$ simulations (thin lines).

galaxy formation process starts at a lower redshift in the former simulation implying a higher mean mass. Such differences are also seen in the stellar particle mass functions (bottom panel in Fig. A.1). Figure 17 compares the galaxy-like object mass function in $G N E$ and $G 1$. Differences can be seen at the low and high-mass ends: at $z=8$ the numerical density of galaxies with a mass higher than $5 \times 10^{6} M_{\odot}$ is $0.2 h^{3} \mathrm{Mpc}^{-3}$ in the $G 1$ simulation but only $0.08 \mathrm{~h}^{3} \mathrm{Mpc}^{-3}$ in GNE. This represents a $60 \%$ decrease in the number of objects in the latter simulation. Moreover the mass of the biggest galaxies is lower in $G N E, 10^{8}$ against $5 \times 10^{8} M_{\odot}$. At $z=6$ differences are less dramatic and only appear for the low-mass end of the galaxy mass function. As discussed in Paper I the influence of nonequipartition processes is dominant in shallow potential wells making gravitational compression unable to heat cosmological plasma to temperatures higher than $10^{6} \mathrm{~K}$. Added to the fact that potential wells become deeper as cosmological evolution proceeds, this explains the decrease in the fraction of out of equilibrium plasma at lower redshift.

The effects on galaxy properties due to thermodynamic modifications of the inter-galactic medium are clearly shown here. A follow-up paper (Courty \& Alimi, in preparation) using the same simulations as in the present paper will discuss changes in the clustering properties of the galaxy-like objects, giving some insights into the physical origin of cosmological bias.

\section{Conclusions}

We examine global galaxy properties and the connection between galaxy formation and the thermodynamics of the cosmological gas in Eulerian hydrodynamical simulations. The dominant processes known to play a role in galaxy formation are included: gravitation, shock heating and cooling processes. The galaxy formation model consists of the identification in the gas distribution of dense and cold regions. A part of this gas is turned into a stellar particle whose collection provides a catalog of galaxy-like objects. In addition, the identification 

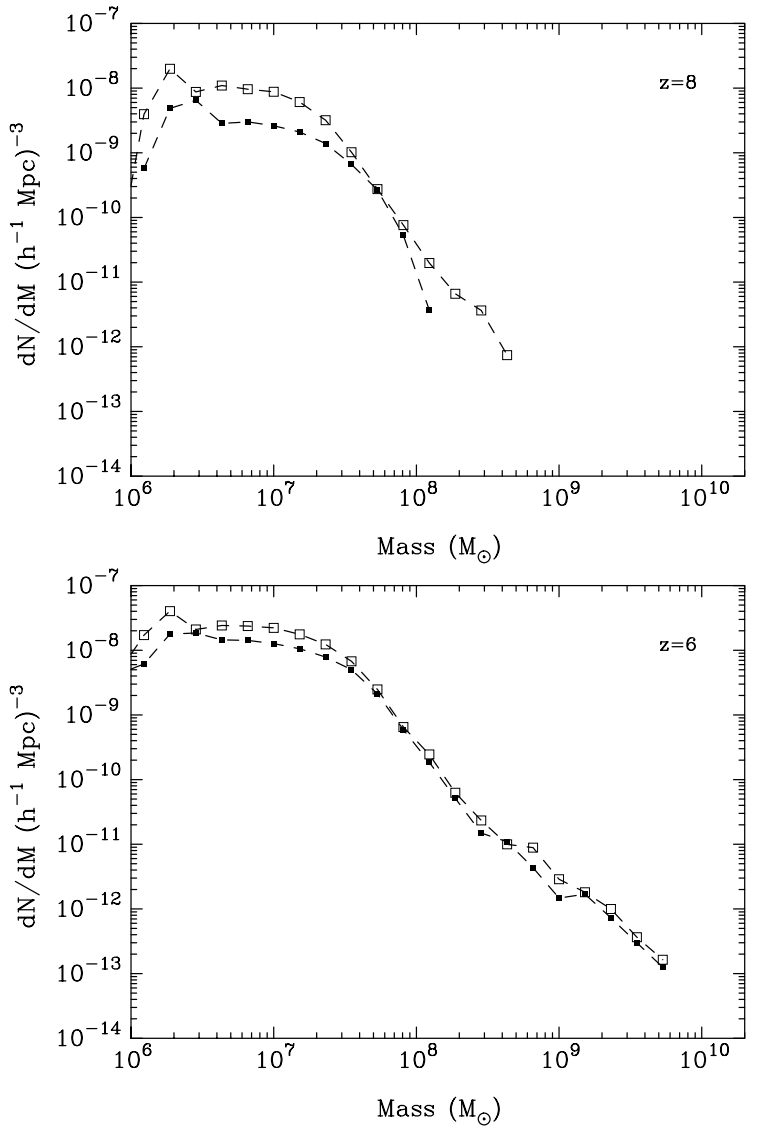

Fig. 17. Galaxy-like object mass function for the GNE (square) and $G 1$ (open square) simulations at $z=8$ and $z=6$.

of dense regions in the dark matter distribution provides a dark matter halo catalog. We estimate a number of properties, the cosmic star formation rate density, the galaxy-like object/dark matter halo mass function and the formation epoch of galaxies.

The most striking result is that considering in the simulations these dominant processes gives galaxy properties consistent with observations. The cosmic star formation rate density shows a peak around $z \sim 3$ and reproduces at low redshift the evolution of the observational star formation rate density, namely the sharp decline between $z=0$ and $z=1$. More than $60 \%$ of the stellar mass has formed by redshift $z=1$. The galaxy-like object mass function presents a significant population of low-mass galaxies and shows an evolution with redshift. Moreover, the galaxy mass function is well described at low redshift in the range $M>10^{8} M_{\odot}$ by an analytical function combining a standard Schechter function with a $\beta$ power-law at the low-mass end. The fit, parameterized by two characteristic masses and two slopes, appears to be in good agreement with the observed galaxy luminosity function. The dark matter halo mass function is found to be well fitted by a three power-law function in the range $10^{9}<M<10^{13} M_{\odot}$ and is steeper than the galaxy mass function in the intermediate mass range. The estimate of the galaxy formation epoch shows that high-mass galaxies form, in the majority, at early epochs. Moreover the galaxy distribution around the mass center of the highest mass dark matter halos shows that these halos include in their center a high-mass, old galaxy-like object. On the other hand the low-mass galaxies present a large dispersion around their conditional mean formation epoch, suggesting that they include recently formed stellar material.

The galaxy formation model is simple enough for the galaxy formation to depend on the thermodynamic properties of the baryonic matter and its distribution. Introducing the photoionization processes has a dominant effect in the low-density regions. This turns into a decrease in the density of the lowest mass objects, $M<10^{7} M_{\odot}$, but no drastic change is seen in the galaxy mass function at the high-mass end, neither in the galaxy formation epoch at intermediate and high mass end, although this quantity shows more dispersion. However these results could depend on the adopted intensity of the ultraviolet background radiation. On the other hand the introduction of the non-equipartition processes between the electrons, ions and neutrals of the cosmological plasma results in a warmer plasma at high redshift and in not too dense regions, than in simulations in which equipartition between species is forced. Hence the longer cooling timescale delays star formation. The galaxy-like object mass function then shows a decrease in the density of objects at the low-mass end.

We also compare two simulations with different computational box lengths and show that galaxy properties depend on the resolution. The amplitudes of the star formation rate density and the galaxy mass function are higher for the simulation with the highest resolution than with the lowest. Indeed adopting a different resolution affects the history of the accretion and gas cooling inside the dark matter potential wells. This results in a change in the fraction of available cold gas at a given time, modifying therefore the galaxy-like object population. We have chosen middle-size box lengths and standard resolutions to allow the formation of high as well as low-mass objects. This work should then be seen as a qualitative analysis of the galaxy formation process and no calibration on observations at $z=0$ is adopted. Altogether the galaxy properties draw a consistent picture of the galaxy formation process and some common features are independent of the resolution: the evolution of the cosmic star formation rate density, the shape of the galaxy-like object mass function, the facts that high-mass galaxies form at early epochs, residing in the highest mass dark matter halos, and that low-mass galaxies, for some of them, form at later epochs, thus showing a star formation activity.

Acknowledgements. Numerical simulations of this paper were performed on NEC-SX5 at the I.D.R.I.S. computing center (France). SC is grateful to A. Hopkins for having kindly provided the observational data of the star formation rate density and to Gunnlaugur Björnsson for a careful reading of the paper. S.C. acknowledges support from a special grant from the Icelandic Research Council. We thank our referee, Naoki Yoshida, for his helpful comments on the manuscript.

\section{References}

Ascasibar, Y., Yepes, G., Gottlöber, S., \& Müller, V. 2002, A\&A, 387, 396

Bardeen, J., Bond, J., Kaiser, N., \& Szalay, A. S. 1986, ApJ, 304, 15

Barkana, R., \& Loeb, A. 2000, ApJ, 539, 20

Binggeli, B., Sandage, A., \& Tammann, G. A. 1988, ARA\&A, 509

Bunn, E. F., \& White, M. 1997, ApJ, 480, 6 
Cen, R. 1992, ApJS, 78, 341

Cole, S., Lacey, C. G., Baugh, C. M., \& Frenk, C. S. 2000, MNRAS, 319, 168

Cole, S., Norberg, P., Baugh, C. M., et al. 2001, MNRAS, 326, 255, (The 2dFGRS Team)

Condon, J. J. 1989, ApJ, 338, 13

Connolly, A. J., Szalay, A. S., Dickinson, M., Subbarao, M. U., \& Brunner, R. J. 1997, ApJ, 486, L11

Courty, S., \& Alimi, J.-M. 2004, A\&A, 416, 875

Cross, N., Driver, S. P., Couch, W., et al. 2001, MNRAS, 324, 825, (The 2dFGRS Team)

Ellis, R. S., Colless, M., Broadhurst, T., Heyl, J., \& Glazebrook, K. 1996, MNRAS, 280, 235

Evrard, A. E., Summers, F. J., \& Davis, M. 1994, ApJ, 422, 11

Flores, H., Hammer, F., Thuan, T. X., et al. 1999, ApJ, 517, 148

Folkes, S., Ronen, S., Price, I., et al. 1999, MNRAS, 308, 459

Fukugita, M., Hogan, C. J., \& Peebles, P. J. E. 1998, ApJ, 503, 518

Gallego, J., Zamorano, J., Aragon-Salamanca, A., \& Rego, M. 1995, ApJ, 455, L1

Gardner, J. P., Sharples, R. M., Frenk, C. S., \& Carrasco, B. E. 1997, ApJ, 480, L99

Glazebrook, K., Peacock, J. A., Miller, L., \& Collins, C. A. 1995, MNRAS, 275, 169

Gnedin, N. Y. 1996, ApJ, 456, 1

Gnedin, N. Y. 2000, ApJ, 535, 530

Guzman, R., Gallego, J., Koo, D. C., et al. 1997, ApJ, 489, 559

Haarsma, D. B., Partridge, R. B., Windhorst, R. A., \& Richards, E. A. 2000, ApJ, 544, 641

Hatton, S., Devriendt, J. E. G., Ninin, S., et al. 2003, MNRAS, 343, 75

Hopkins, A. M., Connolly, A. J., \& Szalay, A. S. 2000, AJ, 120, 2843

Hopkins, A. M., Connolly, A. J., Haarsma, D. B., \& Cram, L. E. 2001, AJ, 122, 288

Hui, L., \& Gnedin, N. Y. 1997, MNRAS, 292, 27

Jenkins, A., Frenk, C. S., White, S. D. M., et al. 2001, MNRAS, 321, 372

Katz, N., Weinberg, D. H., \& Hernquist, L. 1996, ApJS, 105, 19

Kauffmann, G., Colberg, J. M., Diaferio, A., \& White, S. D. M. 1999, MNRAS, 303, 188

Kochanek, C. S., Pahre, M. A., Falco, E. E., et al. 2001, ApJ, 560, 566

Lin, H., Kirshner, R. P., Shectman, S. A., et al. 1996, ApJ, 464, 60

Loveday, J. 1997, ApJ, 489, 29

Loveday, J. 1998, in Proc. of the XVIIIth Moriond astrophysics meeting, Dwarf Galaxies and Cosmology, ed. Thuan et al. (Publ: Éditions Frontièrs)

Loveday, J. 2000, MNRAS, 312, 557

Loveday, J., Peterson, B. A., Efstathiou, G., \& Maddox, S. J. 1992, ApJ, 390, 338

Loveday, J., Tresse, L., \& Maddox, S. 1999, MNRAS, 310, 281
Madgwick, D. S. 2002, MNRAS, 333, 133, (The 2dFGRS Team)

Marzke, R. O., Huchra, J. P., \& Geller, M. J. 1994, ApJ, 428, 43

Marzke, R. O., da Costa, L. N., Pellegrini, P. S., Willmer, C. N. A., \& Geller, M. J. 1998, ApJ, 503, 617

Murali, C., Katz, N., Hernquist, L., Weinberg, D. H., \& Davé, R. 2002, ApJ, 571, 1

Norberg, P., Cole, S., Baugh, C. M., et al. 2002, MNRAS, 336, 907, (The 2dFGRS Team)

Osterbrock, D. E. 1989, Astrophysics of gaseous nebulae and active galactic nuclei (Mill Valley, CA: University Science)

Padmanabhan, T. 1993, Structure formation in the Universe (Cambridge University Press)

Pearce, F. R., Jenkins, A., Frenk, C. S., et al. 2001, MNRAS, 326, 649

Quinn, T., Katz, N., \& Efstathiou, G. 1996, MNRAS, 278, L49

Ratcliffe, A., Shanks, T., Parker, Q. A., \& Fong, R. 1998, MNRAS, 293, 197

Rees, M. J., \& Ostriker, J. P. 1977, MNRAS, 179, 541

Rowan-Robinson, M., Mann, R. G., Oliver, S. J., et al. 1997, MNRAS, 289, 490

Schechter, P. 1976, ApJ, 203, 297

Scott, J., Bechtold, J., Morita, M., Dobrzycki, A., \& Kulkarni, V. P. 2002, ApJ, 571, 665

Serjeant, S., Gruppioni, C., \& Oliver, S. 2002, MNRAS, 330, 621

Silk, J. 1977, ApJ, 211, 638

Somerville, R. S., \& Primack, J. R. 1999, MNRAS, 310, 1087

Spitzer, L. 1978, Physical processes in the interstellar medium (New York: Wiley)

Sprayberry, D., Impey, C. D., Irwin, M. J., \& Bothun, G. D. 1997, ApJ, 482, 104

Springel, V., \& Hernquist, L. 2003, MNRAS, 339, 312

Steidel, C. C., Adelberger, K. L., Giavalisco, M., Dickinson, M., \& Pettini, M. 1999, ApJ, 519, 1

Sugiyama, N. 1995, ApJS, 100, 281

Sullivan, M., Treyer, M. A., Ellis, R. S., et al. 2000, MNRAS, 312, 442

Sunyaev, R. A., \& Zel'dovich, Y. B. 1972, A\&A, 20, 189

Teyssier, R., Chièze, J.-P., \& Alimi, J.-M. 1998, ApJ, 509, 62

Tresse, L., \& Maddox, S. J. 1998, ApJ, 495, 691

Treyer, M. A., Ellis, R. S., Milliard, B., Donas, J., \& Bridges, T. J. 1998, MNRAS, 300, 303

Weinberg, D. H., Hernquist, L., \& Katz, N. 1997, ApJ, 477, 8

Weinberg, D. H., Davé, R., Gardner, J. P., Hernquist, L., \& Katz, N. 1999, in Photometric Redshifts and the Detection of High Redshift Galaxies, ASP Conf. Ser. 191, 341

Weinberg, D. H., Hernquist, L., \& Katz, N. 2002, ApJ, 571, 15

White, S. D. M., \& Rees, M. J. 1978, MNRAS, 183, 341

Yan, L., McCarthy, P. J., Freudling, W., et al. 1999, ApJ, 519, L47

Zucca, E., Zamorani, G., Vettolani, G., et al. 1997, A\&A, 326, 477 


\section{Online Material}


J.-M. Alimi and S. Courty: Thermodynamic evolution of cosmological baryonic gas. II., Online Material p 2
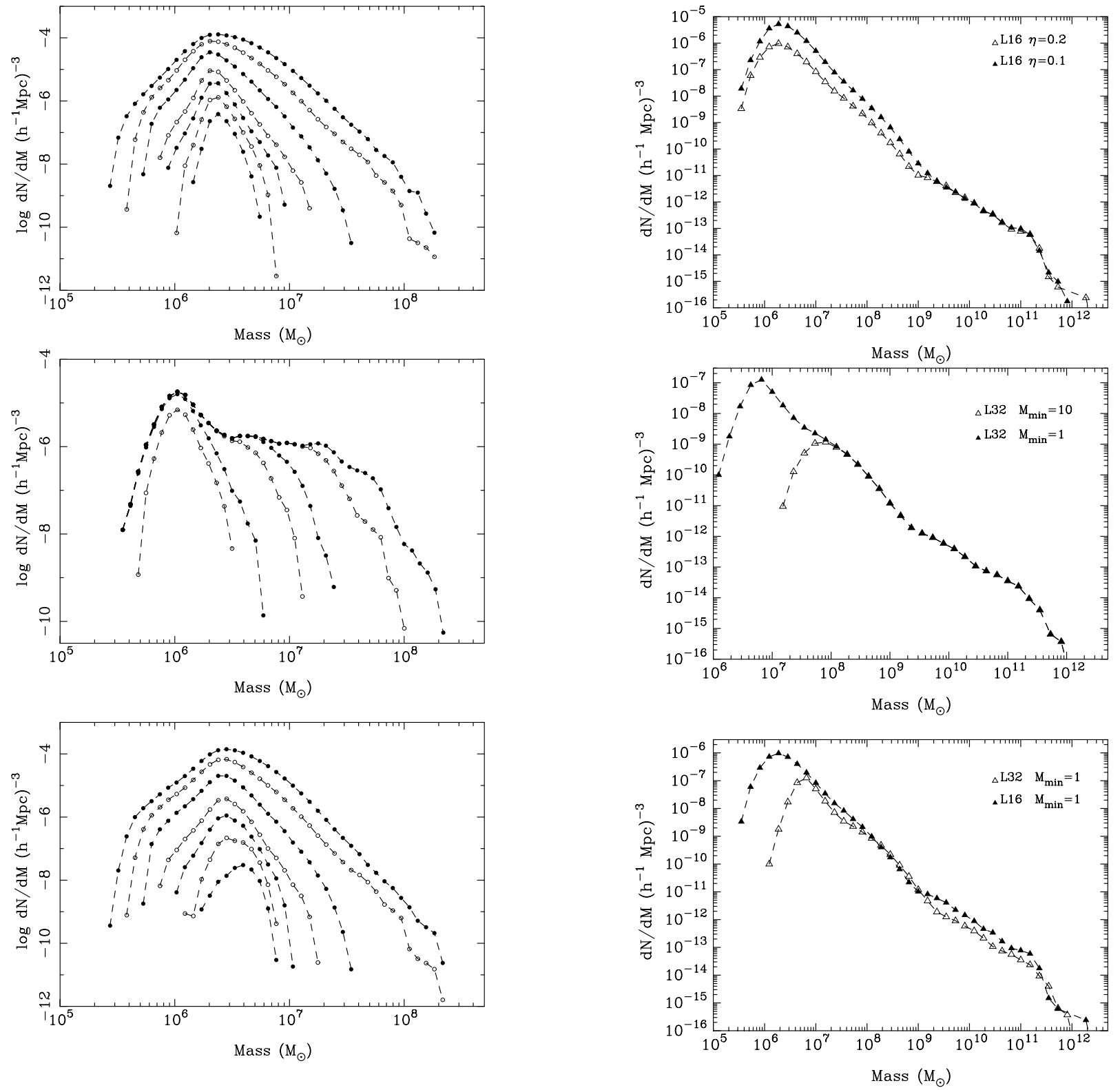

Fig. A.1. Evolution with redshift of the stellar particle mass function for the $G 1, G P$ and $G N E$ simulations (from top to bottom panels, respectively). The mass function is shown at $z=9,8,7,6,4,2,0$ for the $G 1$ and $G N E$ simulations and at $z=8,7,5,4,2,0$ for the $G P$ simulation (alternatively filled and open circles, from bottom to top).

\section{Appendix A: The stellar particle mass function}

Figure A.1 presents the evolution with redshift of the stellar particle mass function in the three kinds of simulations. In $G 1$ the stellar particle mass ranges between $3 \times 10^{5}$ and $2 \times 10^{8} M_{\odot}$ at $z=0$, typically that of the globular clusters. We note that the mass range of the particles increases as the redshift decreases: at any epoch, not only have low-mass stellar particles formed, but also larger ones.

In the $G P$ simulation (middle panel) the formation of the low-mass stellar particles is stopped when photoionization processes become dominant, for redshifts less than 7 (after the epoch of the sharp steepness of the ultraviolet background

Fig. A.2. Galaxy-like object mass function for the $G 0\left(L_{\mathrm{box}}=32\right)$ and $G 1\left(L_{\mathrm{box}}=16\right)$ simulations at $z=0$ computing by considering different values of $\eta$ (top panel) and $M_{\min }$ (middle panel). The bottom panel compares both resolutions.

radiation spectrum, Fig. 1). At lower redshift the mass function presents an evolution for particles with mass higher than $3 \times 10^{6} M_{\odot}$ and the general shape seen in the top panel is modified.

The bottom panel displays the mass functions for the simulation with non-equipartition processes: at high redshift the general shape of upside down "V" seen for the $G 1$ simulation, with a similar maximum (around $2 \times 10^{6} M_{\odot}$ in $G 1$ ) shows that the numerical density of stellar particles is much lower in the $G N E$ simulation than in $G 1$. 


\section{Appendix B: Sensitivity to parameters}

We briefly discuss how the galaxy mass function is sensitive to the parameters involved in determining the galaxy-like objects and to the resolution.

The top panel of Fig. A.2 shows the influence of the link parameter $\eta$ used to group stellar particles and initially fixed to 0.2 (see Sect. 2). Taking $\eta=0.1$ results in a steeper $\beta$ slope. The catalog then includes a larger number of low-mass objects. On the other hand, choosing $\eta=0.3$ would result in a shallower $\beta$ slope. Nevertheless there is no modification in the $\alpha$ power-law range.

The middle panel of Fig. A.2 compares the galaxy mass function at $z=0$ in the $G 0$ simulation considering
$M_{\min }=1$ and $M_{\min }=10$. The parameter $M_{\min }=1$ means that even a single stellar particle is identified as a galaxy-like object. This extends the mass function towards lower mass, shifting the decrease at the low mass end, but does not change the rest of the mass function.

Finally the bottom panel of Fig. A.2 illustrates the influence of the resolution. As already seen with the fits in Fig. 8 the $\alpha$ and $\beta$ slopes and the characteristic mass $M_{*}$ are roughly similar for the two simulations. But in the high-resolution simulation, $G 1$, the transitional mass $M_{\mathrm{t}}$ and the decrease at the low mass end are shifted towards lower mass, this simulation being able to form lower mass objects. 